\title{
Evidence for a shared mechanism used in multiple-object tracking and subitizing
}

\author{
Dana L. Chesney • Harry Haroutioun Haladjian
}

Published online: 4 October 2011

(C) Psychonomic Society, Inc. 2011

\begin{abstract}
It has been proposed that the mechanism that supports the ability to keep track of multiple moving objects also supports subitizing - the ability to quickly and accurately enumerate a small set of objects. To test this hypothesis, we investigated the effects on subitizing when human observers were required to perform a multiple object tracking task and an enumeration task simultaneously. In three experiments, participants (Exp. 1, $N=24$; Exp. 2, $N=11$; Exp. 3, $N=37$ ) enumerated sets of zero to nine squares that were flashed while they tracked zero, two, or four moving discs. The results indicated that the number of items participants could subitize decreased by one for each item they tracked. No such pattern was seen when the enumeration task was paired with an equally difficult, but nonvisual, working memory task. These results suggest that a shared visual mechanism supports multiple object tracking and subitizing.
\end{abstract}

Keywords Object-based attention · Working memory · Dual-task performance

\section{Introduction}

Humans use three types of processes to judge the numerosity of a set: counting, estimating, and subitizing. Counting assesses numerosity by assigning each member of a set

\section{L. Chesney $(\triangle)$}

Department of Psychology, University of Notre Dame,

118 Haggar Hall,

Notre Dame, IN 46556, USA

e-mail: Dana.Chesney.3@nd.edu

H. H. Haladjian

Rutgers Center for Cognitive Science, Rutgers University,

New Brunswick, NJ, USA

e-mail: haroutioun@gmail.com exactly one number label from an ordered count list (Gelman \& Gallistel, 1978). This process is very accurate but relatively slow: The time it takes to count increases linearly with each additional item. Estimating assesses set size quickly without performing a serial count (Kaufman, Lord, Reese, \& Volkmann, 1949). This process is supported by an approximate number system (ANS) that is very fast, but not particularly accurate (Dehaene, Dehaene-Lambertz, \& Cohen, 1998; Gallistel \& Gelman, 1992; Whalen, Gallistel, \& Gelman, 1999). For example, at a glance, one can estimate that there are about 20 seats in a classroom or about 2,000 seats in a stadium. Subitizing also assesses numerosity without performing a serial count. This process is fast (like estimation) and accurate (like counting), but can only enumerate sets of up to four or five items (Atkinson, Campbell, \& Francis, 1976; Feigenson, Dehaene, \& Spelke, 2004; Klahr, 1973; Mandler \& Shebo, 1982; Trick \& Pylyshyn, 1993, 1994b).

Subitizing can be distinguished from counting by the time needed to produce accurate numerosity judgments, and distinguished from estimation by response variability. ANS estimates show scalar variability: The variance in numerosity estimates increases proportionately with set size, resulting in a constant coefficient of variation $(\mathrm{CV})$, so that the ratio of the standard deviation to the mean $(S D / M)$ is constant as set size increases (Gallistel \& Gelman, 2000; Gibbon, 1977; Moyer \& Landauer, 1967; Whalen et al., 1999). A classic indication of subitizing is that the CVs of small-set enumerations are significantly less than are typically seen when estimating, even when conditions preclude counting (Cordes, Gelman, Gallistel, \& Whalen, 2001; Kaufman et al., 1949).

A separate subitizing mechanism?

There is some debate over whether subitizing and estimation are supported by separate mechanisms. Some suggest 
that the mechanism that allows for the fast reaction times and low variability seen in subitizing is in fact the ANS (van Oeffelen \& Vos, 1982; Vetter, Butterworth, \& Bahrami, 2008). Under this view, the subitizing effect is merely a result of ANS representations of neighboring numerosities being more discriminable for values in the small number range. The argument is as follows: ANS representations of large numerosities are more variable, and thus overlap with the representations of many neighboring values, leading to the production of more inexact responses. As numerosity decreases, however, these ANS representations have lower variability, and thus overlap with fewer neighboring values. For small numerosities, ANS representations have sufficiently different distributions from neighboring values that there is virtually no overlap, and thus correct values are consistently produced. Indeed, a formal model of the ANS produces low variability in the subitizing range, if the model represents numerosities as numerical magnitudes with normal variation around the correct value (with an $S D$ equivalent to the exact value times a constant, the $\mathrm{CV}$ ) and it reports values as the positive integer these numerical magnitudes most closely approach. Error rates produced by such a model are illustrated in Fig. 1. This subitizing range is dependent on the $\mathrm{CV}$ of the magnitude representation. Note that the projected end of the subitizing range - marked by an inflection point that indicates the peak acceleration in error rates as numerosities increaseoccurs between 4 and 5 when the $\mathrm{CV}$ of the magnitude representation is .05 , between 2 and 3 when the CV is . 10 , and between 1 and 2 when the CV is .15.

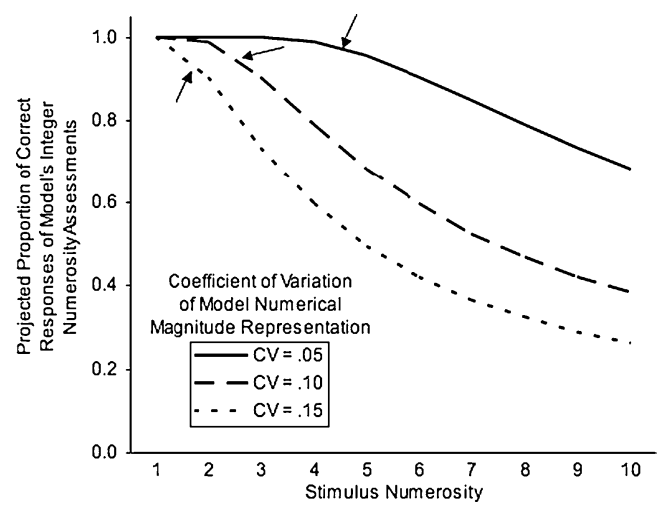

Fig. 1 Expected proportions of correct enumeration reports made by a model approximate number system that represents numerosities as numerical magnitudes with normal variation around the exact correct value and a standard deviation equivalent to the exact value multiplied by a constant (CV, the coefficient of variation); the reported values are the positive integers these numerical magnitudes most closely approach. Values were calculated in MATLAB via the code normedf $(\mathrm{N}+.5, \mathrm{~N}, \mathrm{~N} * \mathrm{CV})$-normcdf $(\mathrm{N}-.5, \mathrm{~N}, \mathrm{~N} * \mathrm{CV})$, where $N$ is the stimulus numerosity and $\mathrm{CV}$ is the coefficient of variation on the numerosity magnitude representation in the accumulator. Arrows indicate inflection points
Studies that have specifically investigated whether subitizing involves a separate process from estimation have yielded somewhat contradictory results. For example, in a study by Revkin, Piazza, Izard, Cohen, and Dehaene (2008) that carefully controlled for the overlap of ANS representations, the authors found that there was indeed particularly low variability in small-set enumeration (1-4 items). Participants in this study named set numerosity in one experiment and identified which of two displays was more numerous in another experiment. The stimulus sets (black dots) were either small (1-8) or were large and presented in "decade quantities" $(10,20, \ldots 80)$. Displays appeared briefly $(150 \mathrm{~ms})$ in order to prevent verbal counting, and total dot area was held constant in half of the trials to prevent the usage of nonnumerosity cues. The authors reasoned that if the same mechanism is used to produce fast enumerations of all numerosities, the CVs seen across the ranges in the smallset and large-set displays should match, since the ratios of the numerosities were equivalent. The results indicated that performance was more accurate in the small than in the large number range - a distinctly low CV was observed for the displays of 1-4 (out of 8) items and not for displays with $10-40$ (out of 80) items. The authors argued that these results indicated that subitizing utilizes a system distinct from estimation. Results by Cordes et al. (2001), however, supported a different conclusion. In this study, participants were tasked to quickly press a button a number of times so as to match the value of a numeral probe. To prevent verbal counting, participants said "the" with every keypress. No differences in the CVs were observed between the small and large number ranges, suggesting that the same system was used to enumerate all numerosities.

Whether or not subitizing in other perceptual modalities utilizes a mechanism separate from the ANS remains uncertain. Nevertheless, it is somewhat telling that studies of subitizing with auditory stimuli (Camos \& Tillmann, 2008) or tactile perception (Ferrand, Riggs, \& Castronovo, 2010; Plaisier, Bergmann Tiest, \& Kappers, 2009; Riggs et al., 2006) have found subitizing ranges of 2 or 3 , as opposed to the 4 or 5 typically found for visual items. Furthermore, some tactile perception subitizing studies have found no evidence of subitizing (Gallace et al., 2006), and another found that subitizing occurs only under certain conditions, such as when a limited number of fingers are stimulated (Plaisier \& Smeets, 2011). These findings, along with those on visual subitizing, suggest that at least some of the basic mechanisms that allow for subitizing are specific to the stimulus modality. Therefore, we confined the scope of this study to one modality: vision. The disparity between the results of Revkin et al. (2008) and Cordes et al. (2001) suggest that there may be a mechanism that allows for the increased accuracy in the enumeration of approximately four visual objects that is not available for the enumeration of 
sequential keypresses. Thus, we find compelling the hypothesis that the mechanism that supports the subitizing of visual sets is part of the visual system.

\section{Subitizing mechanism: various accounts}

One candidate mechanism that may support visual subitizing is the mechanism that underlies multiple object tracking (Feigenson et al., 2004; Pylyshyn, 1989; Trick \& Pylyshyn, 1989, 1994b). Multiple object tracking (MOT) involves the simultaneous visual tracking of several moving items (for a review, see Cavanagh \& Alvarez, 2005; Scholl, 2009). According to Pylyshyn and colleagues, MOT is supported by a visual indexing mechanism that can individuate and "stick to" several items in a visual scene, keeping track of these items as they move (Pylyshyn, 1989, 1994, 2001; Pylyshyn \& Storm, 1988). Furthermore, Trick and Pylyshyn (1993, 1994b) have proposed that indexes are deployed during enumeration to produce the subitizing effect. On this view, an index is automatically assigned to each to-beenumerated object. Subitizing is observed because the numerosity of these indexed items can be determined quickly, primarily because an indexed object is already individuated from a visual scene and has a pointer assigned to it (hence, a search process is not required to locate these objects). These attached indexes can thus be enumerated quickly by a secondary mechanism, such as serial counting or estimation. In support of this secondary-mechanism account of subitizing, it has been noted that the typical subitizing range of 4-5 (Revkin et al., 2008; Trick \& Pylyshyn, 1994b) parallels the typical tracking limit of 4-5 (Pylyshyn, 1989).

Visual indexes act very much like object files, which some suggest support small-set enumeration (Feigenson \& Carey, 2003). Object files are collections of features that are bound together to form working memory representations of particular items in the world (Kahneman, Treisman, \& Gibbs, 1992). It is hypothesized that humans are able to simultaneously individuate and represent up to $\sim 4$ items using these object files. The numerosity of the set of occupied object files could therefore be assessed by a secondary mechanism in which occupied object files are matched by one-to-one correspondence to a set of known quantity (Feigenson \& Carey, 2003). However, an alternative account (see Le Corre \& Carey, 2007) suggests that the "fullness" of one's object files can be directly associated with a specific cardinality, allowing for the rapid assessment of small set numerosity without invoking the ANS or any other mechanism to tally the occupied object files. A similar mapping could be made between cardinalities and the "fullness" of one's visual indexes. This direct-assessment account of subitizing can be considered analogous to judging how many golf balls are in a bag on the basis of how full the bag is. If a limited number of golf balls will fit into the bag-for example, four - one can learn that a half-full bag indicates there are only two golf balls.

Recent findings, however, have led us to doubt whether a direct-assessment method could be successfully implemented using visual indexes. Several studies have suggested that the capacity limit on tracking is not fixed, but fluctuates as a function of a variety of factors, including item separation and item speed (e.g., Franconeri, Jonathan, \& Scimeca, 2010; Horowitz \& Cohen, 2010). This would be particularly problematic to a system under which numerosity was assessed by directly gauging the "fullness" of active indexes. If the number of available indexes (or, in the earlier analogy, the size of the golf ball bag) varies, an indexing mechanism operating at $50 \%$ capacity, for example, might index two items under some circumstances and three items at other times. Thus, simply mapping an amount of index activation to a cardinality would be too imprecise to account for subitizing. In contrast, this possible flexibility in the tracking limit is not problematic under Trick and Pylyshyn's (1993, 1994b) secondary-mechanism account of subitizing. According to that hypothesis, indexes are used to enhance rather than supersede existing enumeration mechanisms. Consequently, the exact mechanism and capacity limit of the system that underlies the MOT system is immaterial. As long as this system can simultaneously individuate and keep track of a set of items, it is capable of supporting subitizing.

\section{Detecting whether MOT and subitizing share resources}

One method of determining whether MOT and subitizing indeed share common resources is via a dual-task experiment. Dual-task experiments often have been used to test whether MOT and other tasks share the same cognitive resources, such as working memory or attention (Allen, McGeorge, Pearson, \& Milne, 2006; Fougnie \& Marois 2009a, b; Tombu \& Seiffert, 2008; Zhang, Xuan, Fu, \& Pylyshyn, 2010). The basic hypothesis of a dual-task design is that if two tasks use common resources, increasing the difficulty of one task should impair performance on the second task when they are performed simultaneously.

Prior dual-task experiments have suggested that interference on or from MOT is modality specific. Allen et al. (2006) found varying levels of interference in a dual-task study in which participants performed a tracking task while also performing a variety of secondary tasks. The secondary tasks included a task in which participants judged whether numbers presented at 1 -s intervals throughout the tracking task were high or low, a similarly designed tone categorization task, an articulatory suppression task, and a spatial finger-tapping task. Although secondary tasks in nonvisual modalities did interfere with tracking (i.e., the tone categorization task), the most interference was caused by the visual/verbal digit categorization task. This indicates 
that modality-specific processes are especially sensitive to dual-task designs involving MOT. This, in turn, suggests that interference related to MOT is attributable to shared resources at the level of the "visuospatial sketchpad." Interestingly, the visuospatial sketchpad has been proposed as being used at a stage in the formation of object files that follows the individuation of items and that requires working memory and central executive functions, such as attention, to bind features to objects (Kahneman et al., 1992). Although Allen et al.'s results indicated that secondary tasks in nonvisual modalities can impair tracking, their participants were still able to successfully track at least three items in these conditions - a minor decrement of tracking capacity.

Different interference patterns on a visual working memory task were seen when the secondary task was either an MOT or verbal working memory task. Fougnie and Marois (2006) found that MOT interfered with recall ability in a visual working memory task that required participants to report the locations and colors of objects presented before the tracking task when tracking ceased. When the tracking load increased, recall accuracy decreased. This interference with visual working memory was not observed when the secondary task involved verbal recall (i.e., remembering a string of two or eight spoken consonants) instead of the MOT task. This again highlights the modality-specific nature of interference that occurs from performing MOT and visual working memory tasks simultaneously.

Having determined that MOT creates specific interference with visual processes, it is important to consider which visual mechanisms MOT tasks may load. To further examine this relationship between visual working memory and attentional resources, Fougnie and Marois (2009a) tested whether a tracking task that was performed during the retention interval of a visual working memory task would disrupt the binding of a conjunction of object features (e.g., [red and square], [yellow and circle]). They found that this binding was disrupted when an attentiondemanding tracking task was performed after the presentation of the to-be-remembered object array. They concluded that this interference was due to the tracking task loading visuospatial attention: Visuospatial attention may assist visual working memory by scanning for the objects during the retention interval in order to refresh and maintain the object features in visual working memory. Since visual indexes would need to be still "pointing" to the objects in order for this refresh process to occur successfully, a simultaneous tracking task would very likely disrupt this process. Similarly, Zhang et al. (2010) tested for interference when MOT was performed during the retention period of either a spatial or a nonspatial working memory task. Participants performed MOT during the retention period of an array of either color-location bindings (spatial condition) or color-shape bindings (nonspatial condition). Results indicated that there was resource competition between the tracking and recall tasks only in the high-load spatial condition (recall performance in the low-load spatial condition was not affected by the tracking task). This effect was specific to spatial memory, since recall was not impaired in nonspatial conditions.

Given the findings above, it seems that the most direct method of manipulating the number of indexes available for subitizing is by manipulating tracking load in a concurrently operated MOT task. As tracking multiple objects requires the use of indexes, this would manipulate the number of indexes available at the target acquisition stage of visual processing (Allen et al., 2006), and thus limit the number of indexes available for subitizing. One concern with such dual-task designs is the difficulty of isolating the initial individuation stage of visual cognition from visual working memory and visuospatial attention. This is especially true because indexes are proposed to lay the groundwork for higher-level visual processes by providing pointers that enable the shifting of attention and by providing the structure for the formation of object file representations in working memory. Both visual attention and indexing, however, employ visual resources. Therefore, such a dualtask design would validly test our hypothesis that MOT and subitizing are both supported by the visual mechanism that enables MOT.

\section{Predictions}

In either the secondary-mechanism or the direct-assessment account of subitizing, when more items are in a to-beenumerated set than the number of available indexes, either a serial counting process must be implemented to achieve an exact count or the unaided ANS must be utilized at the expense of accuracy. If the visual indexes used in MOT are also used in subitizing, the enumeration performance of participants who are asked to perform both tasks simultaneously should vary depending on tracking load. For example, people typically can track four or five items (Pylyshyn, 1989); thus, a person tracking one item should have only three or four remaining indexes available that can be used for subitizing. Likewise, a person tracking two items should have two or three remaining indexes, and so on. Differences in enumeration performance would be particularly pronounced in the small number range, yielding an interaction between the influence of tracking load and the numerosity of to-be-enumerated sets on the variability of numerosity judgments.

The manner in which tracking would be predicted to interfere with subitizing, however, differs between the direct-assessment and secondary-mechanism subitizing hypotheses. If subitizing is achieved strictly via the direct assessment of the "fullness" of one's visual indexes, people 
should overestimate small set numerosities by the number of objects they are tracking. To refer back to the golf ball bag analogy, one would not be able to determine how many golf balls are in a bag by that bag's fullness if the bag also contained ping-pong balls. For example, a person might respond "four" if asked to enumerate a set of two items while simultaneously tracking two items, as their indexes would be "full." Such errors could be avoided if one simply enumerated via counting or estimation when any of one's indexes were otherwise engaged. However, in that case, no subitizing ability should be seen when people are tracking, regardless of how many items are being tracked.

Under the secondary-mechanism hypothesis, only the variability of enumeration responses, not the mean, should be affected by tracking. If subitizing utilizes available visual indexes but numerosity is assessed by an outside mechanism able to distinguish indexed to-be-enumerated items from other indexed items (i.e., tracked items), subitizing should still occur when any visual indexes are free, but the subitizing range should be shortened by the number of tracked items. For example, if a person who has a subitizing range of 4 is tracking two moving objects, his or her subitizing range should be shortened by two. Thus, that person would accurately enumerate sets of two items but would show more enumeration errors assessing sets of three or four items.

In the present study, we set out to examine whether and how the mechanism underlying MOT is involved in subitizing. We utilized a dual-task design that combined MOT with an enumeration task. The dual-task employed in Experiment 1 incorporated not only tracking and nontracking conditions, but also different levels of tracking, allowing us to determine the manner in which subitizing ability varies as a function of tracking load. Participants were required to track zero, two, or four out of eight moving discs and to enumerate a set of zero to nine squares that was presented briefly during this period of disc movement. Experiment 2 replicated this task, gathering enough data from each participant to determine whether the group's results were in fact reflective of individual-level performance. Experiment 3 included an alternative dualtask in which enumeration was performed during a period of verbal working memory retention, as well as another replication of the tracking and enumeration dual-task. This allowed us to compare the resulting interference patterns in order to determine whether the interference pattern seen in the tracking and enumeration dual-task could be attributed solely to an increase in the load on general cognitive resources. Findings from all experiments support our hypothesis that the subitizing of visual items and MOT share a common visual mechanism.

For all of these experiments, we used the divergence and convergence of the CVs of the enumeration estimates in the different load conditions to estimate the subitizing range. Brief presentation times prevented participants from counting. Thus, they could enumerate only via subitizing or estimation. Because estimation is characterized by higher CVs than is subitizing, differences in $\mathrm{CVs}$ at low numerosities would be indicative of participants having subitized in some tracking conditions and estimated in others. These higher CVs should converge at higher numerosities, where subitizing is not effective. As a consequence, the subitizing ranges in the different load conditions can be estimated from the points of divergence and convergence of the enumeration CVs among these conditions. Estimating the subitizing range on the basis of CVs allows us to identify small subitizing ranges better than would estimating on the basis of total enumeration error rates. Estimating the subitizing range from error rates requires finding the set size past which enumeration error rates accelerate. Such a method would not be able to distinguish subitizing ranges less than 2-which would be expected in the high-tracking-load conditions-from subitizing over the length of the tested range, because in neither case would an acceleration in the error rates be expected.

\section{Experiment 1}

We devised a dual-task experiment to investigate the role of visual indexes in small-set enumeration. Participants were asked to track zero, two, or four out of eight identical discs. During the tracking task, a set of zero to nine black squares was flashed briefly on the screen. At the end of each trial, participants were asked to report the number of squares that had been presented. In half of the two-tracking and fourtracking trials, participants identified the tracked targets before reporting numerosity. In the rest of the trials, no tracking reports were requested, and participants were instead asked to report numerosity immediately after the discs stopped moving and disappeared from the screen.

It was critical for the tracking task both to begin before and to extend beyond the presentation of the enumeration stimuli. Otherwise, participants could simply switch all of their indexes to the enumeration task when the squares were presented, effectively eliminating the dual-task aspect of the experiment. By extending the tracking period beyond the presentation of the enumeration stimuli, we ensured that participants would have to continue indexing the tracked discs throughout, not just until, the enumeration period in order to perform the tracking task effectively (Pylyshyn \& Storm, 1988). Thus, it was necessary that we use response variability (which distinguishes subitizing from estimation) rather than reaction time (which distinguishes subitizing from counting) to determine the subitizing range.

Past studies suggested that MOT may require attentional resources (Cavanagh \& Alvarez, 2005; Leonard \& Pylyshyn, 2003; Oksama \& Hyönä, 2004). As counting also requires 
attentional resources (Dehaene, 1992), there could be interference between counting and MOT due to competition for limited attentional resources. However, ANS estimation does not require attention (Burr, Turi, \& Anobile, 2010). To ensure that participants estimated (rather than counted) to-beenumerated sets outside of the subitizing range, enumeration displays were presented very briefly $(50 \mathrm{~ms})$. This method has been used successfully to suppress counting in previous studies, and using such methods produces typical subitizing ranges (Revkin et al., 2008).

Method

\section{Participant}

A group of 24 (8 female) undergraduate students at Rutgers University, New Brunswick, each completed one 45- to 50-min session for course credit.

\section{Apparatus}

The experiment was programmed in MATLAB R2009a using Psychophysics Toolbox version 3.0.8 (Brainard, 1997) and presented on an Apple iMac running OS 10.6. The iMac had a 17-in. color LCD display with a resolution of $1,440 \times 900$ pixels and a refresh rate of $60 \mathrm{~Hz}$. This screen's dimensions were approximately $35^{\circ} \times 22^{\circ}(37 \times 23 \mathrm{~cm})$; the stimuli were restricted to appear within an effective viewing region of approximately $28^{\circ} \times 18^{\circ}$ when participants were seated $\sim 60 \mathrm{~cm}$ from the screen. Responses were collected from a standard keyboard and mouse; participants used the keyboard to initiate each new trial at their own pace.

\section{Stimuli}

Frame rate A total of 20 frames were presented per second $(50 \mathrm{~ms} /$ frame $)$.

Tracking stimuli At the beginning of each trial, eight identical solid white discs appeared at random locations on a solid gray background (RGB values: $[110,110,110])$. These discs were approximately 72 pixels in diameter $\left(\sim 1.8^{\circ}\right.$ viewing angle). The discs moved in a random and unpredictable manner typical of previous MOT studies (e.g., Pylyshyn \& Annan, 2006; Pylyshyn, Haladjian, King, \& Reilly, 2008): Discs moved independently at varying rates averaging 20-160 pixels (approximately $0.5^{\circ}-3.5^{\circ}$ ) per second and were allowed to overlap each other while moving. The discs changed direction when they reached the edge of the viewing region, but deflected at unpredictable angles.

Enumeration stimuli The enumeration stimuli were composed of sets of zero to nine squares that could appear in any nonoverlapping arrangement. The minimum distance between squares was 50 pixels $\left(\sim 1^{\circ}\right.$ of visual angle). The display background became slightly lighter (RGB values: $[135,135,135])$ for the duration of the enumeration stimulus presentation. Squares size was controlled such that the surface areas summed either to $30,000,40,000$, 50,000 , or 60,000 pixels, regardless of the numerosity of the sets. The size of individual squares varied both within and across trials. The minimum square size was $50 \times 50$ pixels, and square widths ranged between $\sim 1^{\circ}$ and $\sim 6^{\circ}$ of visual angle. Each square numerosity (excluding zero) was represented an equal number of times by each of the total area options. This ensured that numerosity judgments were not based on continuous display features, such as total screen coverage by the black texture.

\section{General procedure}

Participants sat $\sim 60 \mathrm{~cm}$ from the test display; no restraints were used to restrict head or eye movements. Neither eye movements nor foveation are necessary to subitizing (Trick \& Pylyshyn, 1994a), and one does not need to maintain focus on an item in order to track it, or else MOT would not be possible. Thus, no additional controls for eye movements were used. The experimenter told the participants that they were to keep track of zero, two, or four target discs as the discs moved on the screen and that, during this movement, the participants should assess how many squares were flashed on the screen ("There could be a lot of squares, there could be a few squares, there could be no squares"). They were told that sometimes they would be asked to indicate which items they tracked, and that they always would be asked to indicate how many squares were presented. The participants were asked to complete both tasks as accurately as possible. The experimenter then administered six practice trials to ensure understanding of the tasks.

Each trial began with an initial gray screen with eight identical white discs in a random configuration. A white fixation cross was displayed in the center of the screen to encourage participants to remain focused on this position. At this point, any to-be-tracked discs were identified by flashing the discs white and blue (RGB values: $[0,0,255]$ ) ten times over a 3,000-ms period ( 60 frames, 6 frames per flash). When flashing ceased, the objects began moving randomly around the screen for 6,000 ms (120 frames). At a random point between $750 \mathrm{~ms}$ ( 15 frames) and $5,250 \mathrm{~ms}$ (105 frames) of movement, the enumeration stimuli were presented for $50 \mathrm{~ms}$ ( 1 frame). The discs continued moving for the remainder of the movement period. This final segment of the MOT served as a mask for the enumeration stimuli, preventing counting from afterimages on the display. In a random $50 \%$ of the two- and four-target trials, 
the participants were prompted to select the tracked targets with the mouse pointer. In the rest of the trials, this tracking report was skipped. Next, the discs disappeared, and participants were prompted to use the number keys at the top of the keyboard to indicate how many squares had been presented. Once an entry was made, participants were prompted to press the space bar to initiate the next trial. Participants could choose to take a break at any point during the experiment by waiting to press the space bar. A schematic of this procedure is displayed in Fig. 2. Each session included 200 trials, with 40 trials in each of the five tracking/report conditions (see Table 1). Each of the four possible surface areas described above (30,000 pixels, etc.) was used once for each enumeration stimulus numerosity (one to nine squares) for each tracking/report condition, with an additional four trials in which zero squares were presented.

\section{Results and discussion}

The results of this experiment support our hypothesis that the subitizing of visual items and MOT share a common visual mechanism. The discussion of our results is divided into four parts: (1) We establish that participants both tracked and enumerated in this task, with high tracking performance in all enumeration conditions. (2) We establish that the hypothesis that people subitize by assessing the "fullness" of their visual indexes was not supported. (3) We show that in accordance with the secondary-mechanism hypothesis, the subitizing range decreased as tracking load increased. (4) Finally, we discuss some caveats and concerns, which we address in subsequent experiments. (Note: Here and throughout the article, we include in our analyses and graphs only enumeration reports from trials in which tracking reports were not collected, to reduce any possible interference from tracking response.)

\section{Participants could enumerate while tracking}

Participants tracked quite successfully in this dual-task experiment (see Fig. 3). Tracking targets were all correctly identified in $85 \%$ of the trials with tracking reports in the twotracking condition (chance of guessing all targets on any given trial $=3.6 \%$, since this is equivalent to sampling without replacement; see Hulleman, 2005) and $71 \%$ of such trials in the four-tracking condition (chance of guessing all targets $=1.4 \%$ ). These are typical single-task tracking levels, as seen in prior studies (e.g., Alvarez, Horowitz, Arsenio, Dimase, \& Wolfe, 2005). Each participant performed significantly above chance in both conditions, with rates of perfect tracking ranging from $48 \%$ to $98 \%$ in the twotracking condition and from $33 \%$ to $93 \%$ in the four-tracking condition. A linear regression found that while there did appear to be some decrement in the rates of perfect tracking in the two-tracking condition as the numerosity of the enumeration stimuli increased, the effect size was very small (slope $=-.016, S E=.004 ; t=-4.0, p<.001, R^{2}=.017$ ). Therefore, we concluded that tracking was at or near maximum performance throughout the experiment, and any dual-task trade-offs should be reflected in the subitizing range (see Alvarez et al., 2005).

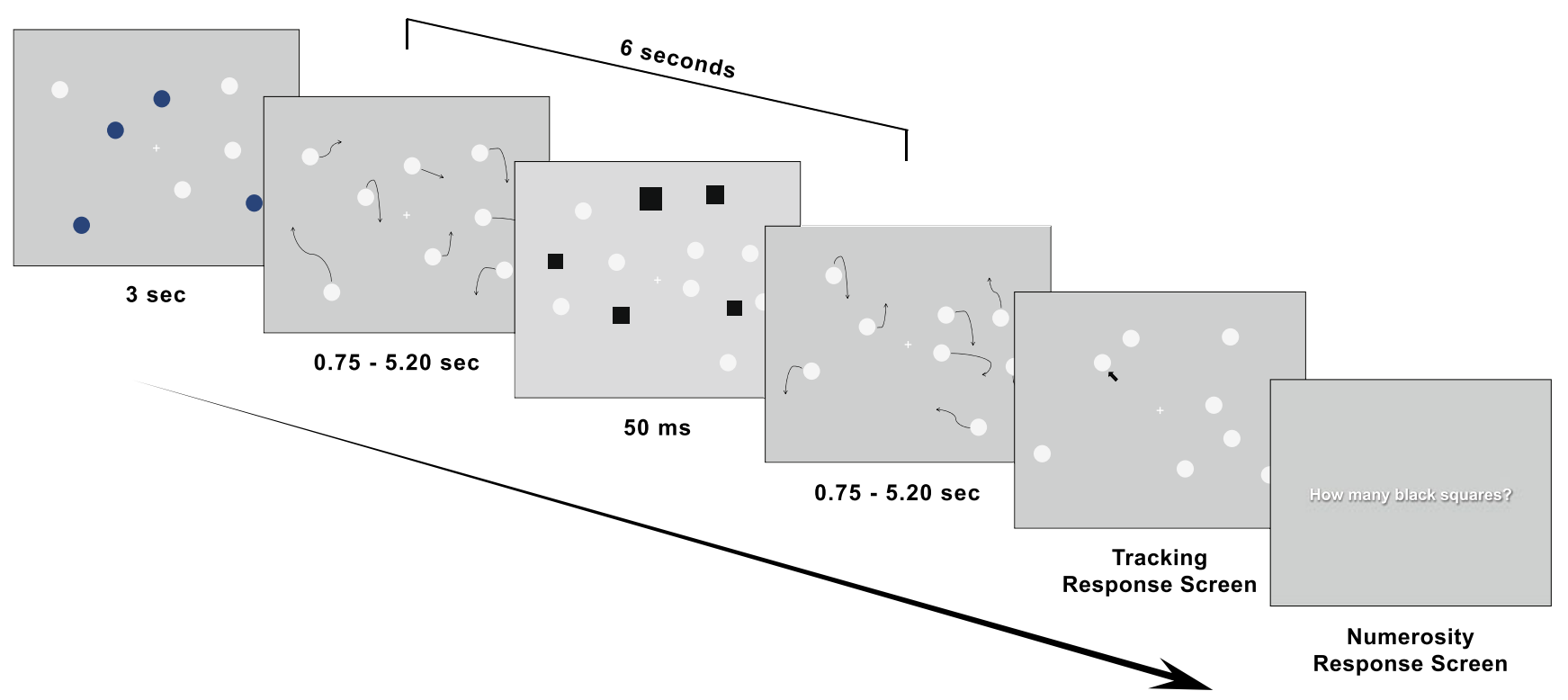

Fig. 2 Schematic of the dual-task experimental design (Experiment 1). Panel 1 depicts the initial screen with targets flashing blue (3 s). Panel 2 depicts random movement of the MOT objects, as indicated by the arrows. Panel 3 depicts the presentation of the enumeration stimuli (black squares) during object movement. Panel 4 depicts the remainder of the movement of the MOT objects. In a random $50 \%$ of the tracking trials, a tracking response screen followed, in which the participant used the mouse to select the target objects (panel 5). Finally, in every trial the participant entered the number of squares that had been flashed, using a standard computer keyboard (panel 6) 
Table 1 Distribution of experimental conditions in Experiment 1

\begin{tabular}{lll}
\hline Items Tracked & With Tracking Report & No Tracking Report \\
\hline 0 targets & n/a & $20 \%(40$ trials $)$ \\
2 targets & $20 \%(40$ trials $)$ & $20 \%(40$ trials $)$ \\
4 targets & $20 \%(40$ trials $)$ & $20 \%(40$ trials $)$ \\
\hline
\end{tabular}

Participants also enumerated successfully while tracking, as can be seen in Figs. 4 and 5. A linear regression showed that, as would be expected, the mean enumeration responses increased with the numerosity of the enumeration stimuli. On average, numerical responses increased by 0.76 $(S E=0.008, t=91.4, p<.001$; intercept $=0.73, S E=0.045$, $\left.t=16.4, p<.001, R^{2}=.744\right)$ for every square presented. Increases were observed for every participant, with coefficients ranging from $.420(S E=.055, t=7.7, p<.001$; intercept $\left.=2.042, S E=0.292, t=7.0, p<.001, R^{2}=.333\right)$ to $.915(S E=.024, t=38.9, p<.001$; intercept $=0.333, S E=$ $\left.0.126, t=2.6, p<.01, R^{2}=.928\right)$.

\section{Testing the direct-assessment hypothesis}

The results do not support the hypothesis that subitizing occurs by direct assessment of the "fullness" of one's indexes, because we did not observe a systematic overestimation of set size by the number of items being tracked. An overestimation by a value approaching 1 for each tracked item would have been expected if this were the case. When analyzing trials in which one to four squares were presented (the normal subitizing range), no significant correlation was found between tracking load and enumeration responses overall $(r=.047, p=.112)$. One of the participants did show a significant correlation $(r=.350, p<.02)$, but this significance was lost after adjusting for the increased

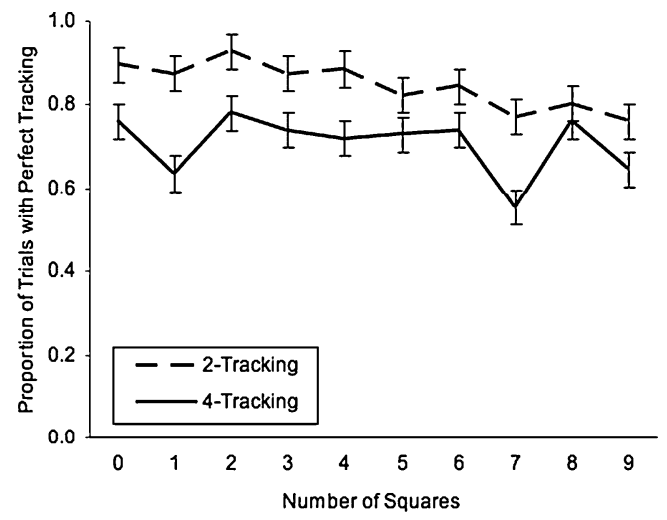

Fig. 3 Experiment 1: Proportions of trials with perfect tracking reports as a function of tracking condition and the number of squares displayed. Error bars represent standard errors
Type I error rate produced from running 24 separate analyses. Furthermore, as discussed below, participants showed some subitizing ability when tracking two items. This serves as evidence against the alternative hypothesis that people can subitize by direct assessment, but do so only when all of their indexes are otherwise unoccupied.

\section{Testing the secondary-mechanism hypothesis}

Because the short presentation time used in this experiment suppressed counting (Revkin et al., 2008), participants had to make numerosity judgments by either subitizing or estimating. Subitizing is characterized by a lower CV than estimation (Whalen et al., 1999). Thus, to determine when participants were subitizing, we calculated each participant's CV $(S D / M)$ for every cell in the design. We then performed a 3 (tracking condition: zero, two, or four items) $\times 6$ (enumeration condition: two to seven squares presented) repeated measures ANOVA on these values. Only the cases of two to seven squares were included in order to control for anchor effects. It has long been observed (Jevons, 1871) that when participants are required to give enumeration responses to stimuli with a fixed range, the responses tend to drift toward the center of that range. The resulting distortion is more pronounced for stimuli at the edges of this range. The influence of these anchor effects can be seen in Fig. 4 in the particularly low mean responses to set sizes 8 and 9, and in the "bow tie" pattern of the standard deviation of these responses, which has its narrowest point for set sizes of 2 (as opposed to 1, which would be expected from scalar variability). Thus, as planned, trials in which zero, one, eight, or nine squares were presented were excluded from our analysis. We found a significant main effect of tracking condition $[F(2,46)=$ 6.3, $\left.p<.005, \eta_{p}^{2}=.214\right]$ and a marginally significant interaction between enumeration condition and tracking condition $\left[F(10,230)=1.7, p<.08, \eta_{p}^{2}=.070\right]$ (see Fig. 6). These results suggest that MOT and subitizing share common resources. When assessing numerosities in the small number range, participants' CVs were higher when tracking load was higher. CVs in different tracking conditions converged at higher numerosities. This indicates that a more variable enumeration process is implemented in the small number range when indexes are busy tracking moving objects.

The hypothesis that subitizing and MOT use a common mechanism is further supported by the subitizing ranges estimated for the different tracking conditions. Subitizing ranges were estimated based on the divergence and convergence of the enumeration CVs, as determined from the overlap of the standard error intervals (see Fig. 6). The subitizing range in the four-tracking condition was estimated as the value 


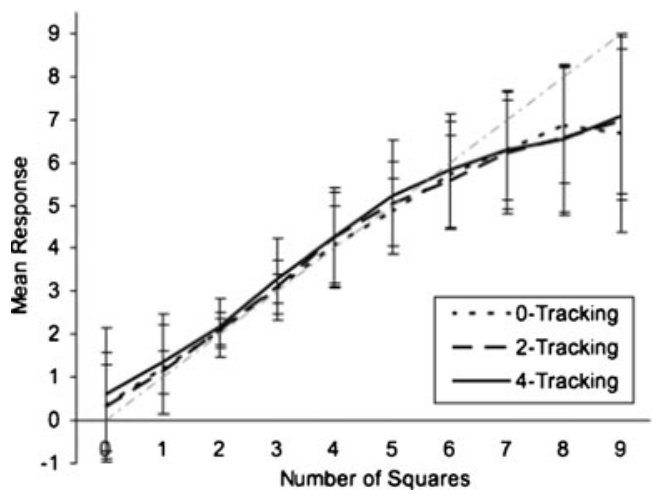

Fig. 4 Experiment 1: Mean enumeration reports as a function of tracking condition and the number of squares displayed. Error bars represent standard deviations. The gray dash-dotted line indicates the correct numerosity

before which its CVs initially diverged from the CVs of the zero-tracking condition. The $\mathrm{CVs}$ of the fourtracking condition were already divergent from the $\mathrm{CVs}$ of the zero-tracking condition when evaluating sets of numerosity 2, and therefore the four-tracking condition was estimated to have a subitizing range of 0.5 . (Note: The range of 0.5 was assigned because it is not possible to distinguish between subitizing ranges of 0 or 1 by this method.) The subitizing range in the twotracking condition was estimated to be 2.5: the average of the numerosity before which its CVs initially diverged from the CVs of the zero-tracking condition and the numerosity before which its CVs initially converged with the CVs of the four-tracking condition. The subitizing range in the zero-tracking condition was estimated to be 3 , the last numerosity before which its CVs reconverged with the CVs of the two-tracking condition.

Confirming these estimates of the subitizing range, decreases in the proportions of trials with correct

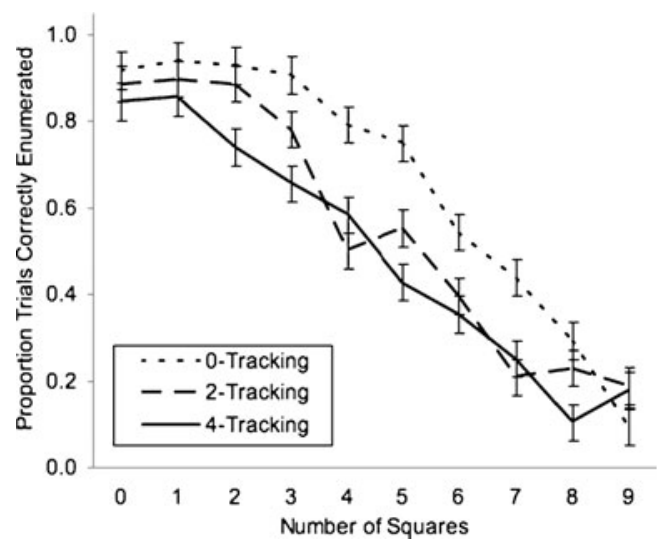

Fig. 5 Experiment 1: Proportions of trials with correct enumeration reports as a function of tracking condition and the number of squares displayed. Error bars represent standard errors

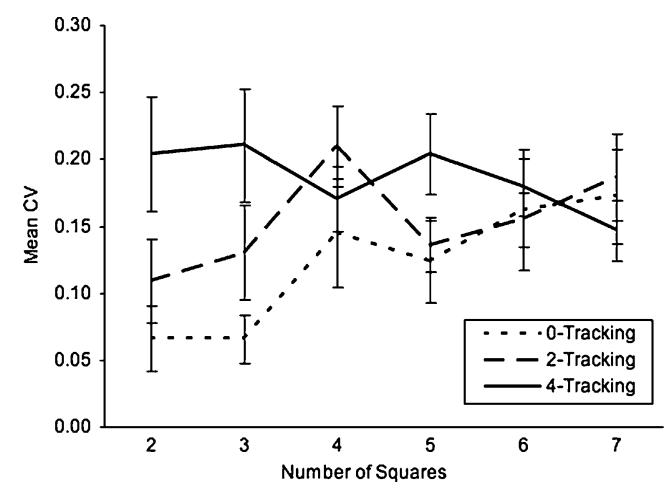

Fig. 6 Experiment 1: Mean coefficients of variation (CVs) of enumeration as a function of tracking condition and the number of squares displayed. Error bars represent standard errors

enumeration responses can be observed at similar numerosities in Fig. 5. In the zero-tracking condition, there is a sharp decline in enumeration performance that indicates a subitizing range of about 3 , after which the proportion of correctly enumerated trials drops more rapidly. A similar decline can be observed in the twotracking condition after 2 or 3 , suggesting a subitizing range of about 2 or 3 items. This decline occurs after 1 in the four-tracking condition, suggesting a subitizing range of about 0 or 1 . (The subitizing ranges of 1 or $0-$ a total lack of subitizing - cannot be distinguished in this manner, as both would show steep drops in accuracy after 1. The enumeration condition with a set size of 0 is a special case, since instead of tallying items that are present, the observer must rather note that there are no items present to be tallied.) It indeed appears that the subitizing range decreased as tracking load increased.

\section{Caveats and concerns}

One concern for interpreting the results from Experiment 1 is that by averaging the participants' data we might be masking individual differences. If the average data are not indicative of individual participants' performance, this might lead to erroneous conclusions on how the MOT and subitizing tasks interact. We addressed this concern in Experiment 2 by gathering sufficient data from each participant (i.e., 40 trials per experimental condition) to determine whether individual performance was consistent with the aggregate pattern. A second concern is that in Experiment 1, it was possible for the randomly placed squares in the enumeration stimuli to overlay the moving discs. This might have increased the variance of the numerosity responses, due to variations in inhibition between empty space and tracked and untracked discs (Pylyshyn et al., 2008). Thus, in Experiment 2, the squares were constrained to appear only in empty space. 


\section{Experiment 2}

Method

Participants

A group of 11 participants (7 female) each completed eight 55 - to 60 -min sessions for a payment of $\$ 10$ per session. Nine were undergraduate students recruited from Rutgers University, and 2 were the authors (who did not receive payment).

\section{Apparatus}

The experiments were programmed in MATLAB R2009a using Psychophysics Toolbox version 3.0.8 (Brainard, 1997) and presented using a Dell Dimension 4550 desktop computer running Windows XP (Intel Pentium 4 processor) and an Apple iMac running OS 10.6. On the Windows computer, the stimuli were displayed on a 19-in. color CRT monitor with a resolution of $1,280 \times 1,024$ pixels and a refresh rate of $60 \mathrm{~Hz}$; the screen dimensions were approximately $34^{\circ} \times 25^{\circ}(35.2 \times 26.4 \mathrm{~cm})$. The iMac's screen had the same properties as the one used in Experiment 1. The stimuli were presented within an effective viewing region of approximately $27^{\circ} \times 20^{\circ}$ (Windows) or $28^{\circ} \times 18^{\circ}$ (iMac) when participants were seated $\sim 60 \mathrm{~cm}$ from the screen; no restraints were used to restrict head or eye movements. Responses were collected from a standard keyboard and mouse; participants used the keyboard to initiate each new trial at their own pace.

\section{Stimuli}

On both computers, the visual appearance of the stimuli was essentially identical to that of the stimuli in Experiment 1. The timing, however, varied between the two systems. As in Experiment 1, participants using the iMac computers $(N=7)$ saw displays presented at 20 frames per second (longer), yielding 3,000 ms of target identification (60 frames) and $6,000 \mathrm{~ms}$ of disc motion (120 frames), with the enumeration stimuli presented for $50 \mathrm{~ms}$ ( 1 frame) at a random point between $750 \mathrm{~ms}$ (15 frames) and 5,250 ms (105 frames) of movement. However, for participants on the Windows computer $(N=4)$, the frame rate was 30 frames per second $(33.3 \mathrm{~ms} /$ frame, shorter $)$. This yielded $2,000 \mathrm{~ms}$ of target identification (60 frames) and 4,000 ms of disc motion (120 frames) at speeds varying between 30 and 240 pixels (approximately $0.75^{\circ}-6^{\circ}$ ) per second, with the enumeration stimuli presented for $33.3 \mathrm{~ms}$ ( 1 frame) at a random point between $500 \mathrm{~ms}$ ( 15 frames) and 3,500 ms (105 frames) of movement. The enumeration stimuli were presented in the same manner as in Experiment 1, with two exceptions. In Experiment 2, five (rather than four) different total surface areas were used when constructing the squares (enumeration stimuli): $30,000,40,000,50,000,60,000$, or 70,000 pixels. Additionally, the squares were constrained to appear only in empty space.

\section{Procedure}

The procedure was identical to that of Experiment 1, except in the following respects: Each of the five (rather than four) possible surface areas was used once per session for each numerosity (one to nine squares) in each of the five tracking/report conditions, with an additional five trials in which zero squares were presented. This yielded a total of 250 trials per session, with 50 trials in each of the tracking/ report conditions. Each participant completed eight sessions, for a total of 2,000 trials. The six practice trials described in Experiment 1 were only given before the first session. No more than two sessions were completed on the same day.

\section{Results and discussion}

The results of this experiment again supported our hypothesis that the subitizing of visual items and MOT share a common mechanism. The discussion of our results is divided into six parts: (1) We establish that participants both tracked and enumerated in this task, with high tracking performance in all enumeration conditions. (2) We show that the directassessment hypothesis was not supported. (3) We show that in accordance with our secondary-mechanism hypothesis, the observed subitizing range decreased as participants tracked more items. (4) We show that this finding in the group data is consistent with individual participants' performance. (5) Finally, we discuss remaining caveats and concerns, which we addressed in the subsequent experiment. Again, we only present enumeration results from the trials in which tracking responses were not collected. In preliminary analyses, we found no effect of the shorter- and longer-duration conditions, and thus the subsequent analyses were collapsed across these conditions.

\section{Participants could enumerate while tracking}

As in Experiment 1, participants tracked successfully in this dual-task (see Fig. 7). Overall, tracking performance was high, with the proportion of trials with perfect tracking at $90 \%$ in the two-tracking condition (chance of guessing all items = $3.6 \%$ ) and $79 \%$ in the four-tracking condition (chance of guessing all items $=1.4 \%$ ), typical single-task tracking levels seen in prior studies (e.g., Alvarez et al., 2005). Each participant performed significantly above chance in both conditions, with the proportion of trials with perfect tracking ranging from $66 \%$ to $99 \%$ in the two-tracking condition and from $45 \%$ to $92 \%$ in the four-tracking condition. While there 
was some decrement in perfect-tracking rates in both the twotracking and four-tracking conditions as the numerosity of the enumeration stimuli increased, this effect size was very small (two tracking, slope $=-.004, S E=.002, t=-2.5, p<.02, R^{2}=$ .001 ; four-tracking, slope $=-.009, S E=.002, t=-4.2, p<$ $\left..001, R^{2}=.004\right)$. Therefore, we concluded that tracking performance was at or near maximum throughout this experiment, and any dual-task trade-offs should be seen in the subitizing range.

Again, participants enumerated successfully during the tracking task (see Figs. 8 and 9). A linear regression showed that on average, numerical responses increased by $0.833(S E=0.003, t=244.9, p<.001$; intercept $=0.439$, $\left.S E=0.018, t=24.2, p<.001, R^{2}=.820\right)$ for every square presented. An increase was observed for every participant, with coefficients ranging from $.672(S E=.017, t=40.7$, $p<.001$; intercept $=1.05, S E=0.088, t=11.9, p<.001$, $\left.R^{2}=.589\right)$ to $.935(S E=.007, t=125.9, p<.001$; intercept $=$ $0.190, S E=0.04, t=4.8, p<.001)$.

\section{Testing the direct-assessment hypothesis}

To investigate whether subitizing occurs by judging the "fullness" of the indexing mechanism, we again correlated the value of the participants' enumeration reports with the number of items that they were tracking while making these numerosity judgments. When analyzing trials in which one to four squares were presented, a direct correlation was found between tracking load and the participants' numerical responses $(r=.040, p<.005)$. This correlation was also significant for 1 participant $(r=.137, p<.005)$. A linear regression found that, on average, numerical responses increased by $0.031(S E=0.011, t=2.9, p<.005$; intercept $=$ 2.458, $S E=.028, t=89.1, p<.001, R^{2}=.002$ ) for every item tracked. For the participant with a significant correlation, this coefficient was $0.123(S E=0.041, t=3.0, p<$ .005 ; intercept $=2.428, S E=0.106, t=23.0, p<.001, R^{2}=$

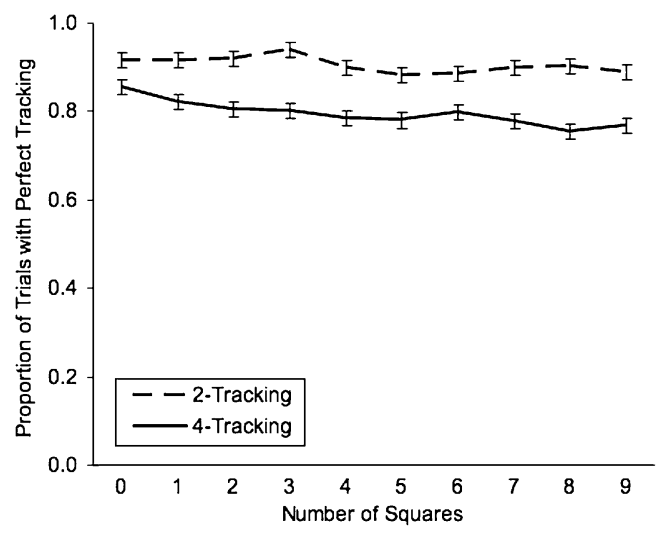

Fig. 7 Experiment 2: Proportions of trials with perfect tracking reports, as a function of tracking condition and the number of squares displayed. Error bars represent standard errors

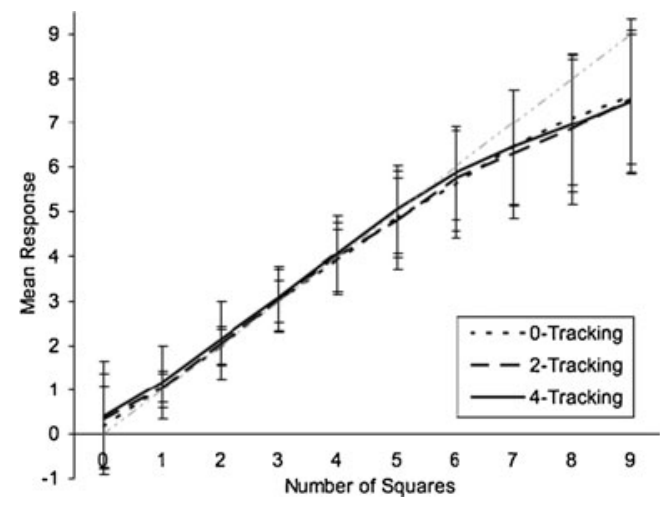

Fig. 8 Experiment 2: Mean enumeration reports as a function of tracking condition and the number of squares displayed. Error bars represent standard deviations. The gray dash-dotted line indicates the correct numerosity

.019). Thus, each extra item tracked only increased the reported numerosity of small sets by 0.031 on average, and 0.123 for 1 individual. These slopes and effect sizes are quite small, and as such do not support the conclusion that cardinalities subitized from small sets increase in one-to-one correspondence with the number of items tracked. Furthermore, as discussed below, participants showed some subitizing ability when tracking two items. Again, this serves as evidence against the alternative hypothesis that people subitize via direct assessment, but only when all of their indexes are unassigned.

\section{Testing the secondary-mechanism hypothesis}

Once again, we estimated subitizing ranges in the different tracking load conditions on the basis of the CVs of the enumeration responses. To analyze the CVs while still allowing each trial to be included separately in the analyses, we first calculated each participant's mean numerical response for a particular kind of trial - that is, trials in which the same number of squares were presented and the same number of

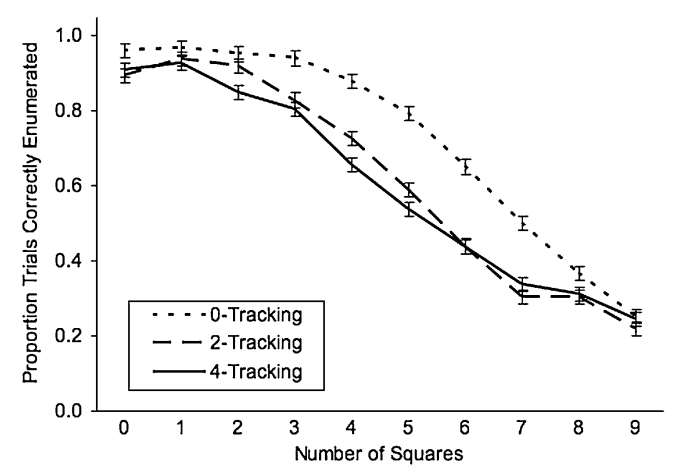

Fig. 9 Experiment 2: Proportions of trials with correct enumeration reports as a function of tracking condition and the number of squares displayed. Error bars represent standard errors 
items were tracked. We then found the absolute difference between this mean value and the participant's actual responses for every trial. We then divided these values by those individual cell means. Thus, the formula used to find these mean adjusted variance measures was (|Response - Cell Mean| / Cell Mean). This serves as an analogue of the CV that allows for independent evaluations of each trial (i.e., difference $/ M$, as opposed to $S D / M$ ).

As before, we only included cases in which two to seven squares were presented in our analysis (see Experiment 1 for discussion). Thus, we ran a 3 (tracking condition: zero, two, or four items) $\times 6$ (enumeration condition: two to seven squares presented) $\times 11$ (participants) mixed-model ANOVA on these mean adjusted variance measures, where tracking condition and enumeration condition were included as fixed factors and the participant ID was included as a random factor. A significant effect of participant was to be expected, since not only are some participants simply more variable than others, but also because of participants' tendency to have different "favorite numbers." Often, participants showed a preference for responding with certain specific values, such as "five" or "seven." This led to individuals showing somewhat deceptive levels of accuracy when assessing cardinalities corresponding to these "favorite numbers." Including participant ID as a random variable allowed us to take these effects into account when assessing the influences of both the tracking and enumeration conditions on the variability of the participants' performance.

We found significant main effects for tracking condition $\left[F(2,20)=22.8, p<.001, \eta_{p}^{2}=.696\right]$, enumeration condition $\left[F(5,50)=6.2, p<.001, \eta_{p}^{2}=.385\right]$, and participant $\left[F(10,3.14)=55.0, p<.005, \eta_{p}^{2}=.994\right]$. Additionally, there was a significant interaction between enumeration condition and tracking condition $[F(10,100)=$ $\left.2.0, p<.05, \eta_{p}^{2}=.170\right]$ and a three-way interaction between enumeration condition, tracking condition, and participant $\left[F(100,7722)=3.6, p<.001, \eta_{p}^{2}=.045\right]$. Our mean adjusted variance measure typically increased with both the number of tracked items and the number of squares presented (see Fig. 10), though there was variation among participants as to where and how this occurred (see Table 2). When participants tracked more items, their enumerations of small numerosities were typically more variable. However, these variability levels tended to converge as the numerosity of the enumeration stimuli increased. These results replicated the findings from Experiment 1, indicating that MOT and subitizing share common resources.

Furthermore, the subitizing range again decreased as the number of objects tracked increased. As in Experiment 1, we estimated the subitizing range via the divergence and convergence of the variance measures in the different tracking conditions. The subitizing range was estimated to

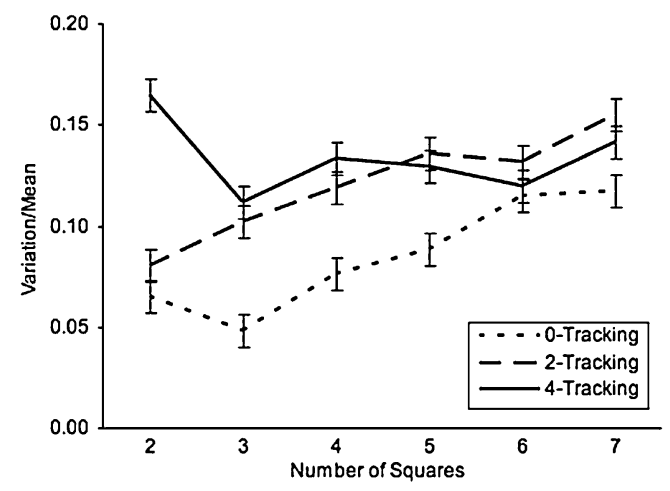

Fig. 10 Experiment 2: Mean-adjusted variation of enumeration reports (average distances from cell means divided by the cell means), as a function of tracking condition and the number of squares displayed. Error bars represent standard errors

be 5 in the zero-tracking condition, 2 in the two-tracking condition, and 0.5 in the four-tracking condition (see Fig. 10). Confirming these estimates, drops in correct enumeration rates can be observed at similar values on Fig. 9. In the zero-tracking condition, there was a general decline in the rate of correct enumeration responses that seems to indicate a subitizing range of about 3 or 4 . Similar declines can be observed in the two-tracking condition after 2 , and in the four-tracking condition after 1 , which suggest subitizing ranges of about 2 and about 0 or 1, respectively. These findings further support the hypothesis that subitizing and MOT use a common visual mechanism.

\section{Individual performance is consistent with group performance}

The issue of "favorite numbers" previously discussed makes it difficult to use the methods described above to determine how tracking influenced individuals' subitizing ranges. However, according to our hypothesis, increased tracking load results in less subitizing in the small number range, and consequently more variability when enumerating small sets. Therefore, the correlation between the meanadjusted variation of participants' enumeration reports and the numerosity of the enumeration stimuli should be less positive when participants were tracking more items. Indeed, looking at enumeration reports from trials in which two to seven squares were presented in the four-tracking condition for the group as a whole, our variance measure was not correlated with the numerosity of the enumeration stimuli $(r=-.023, p=.176)$, but there was a direct correlation between our variance measure and the numerosities of the enumeration stimuli in the two-tracking condition $(r=.133, p<.001)$ and the zero-tracking condition $(r=.136, p<.001)$. Correlations for individual participants were typically consistent with this pattern, as can be seen in Table 2 . 
Table 2 Experiment 2: Individual participants' correlations between mean-adjusted variation in enumerate and: the number of tracked items (column 2), the number of squares presented (column 3 ), and the number of squares presented for each tracking condition (columns 4, 5, and 6)

These analyses only include cases in which two to seven squares were presented. SS\# refers to participant number. ${ }^{*} p<.05 .{ }^{* *} p<.01 .{ }^{* * *} p<.001$

\begin{tabular}{llllll}
\hline SS\# & Items Tracked & Number of Squares & \multicolumn{2}{l}{ Number of Squares, in Conditions When Tracking } \\
\cline { 3 - 5 } & & & 0 Items & 2 Items & 4 Items \\
\hline 201 & $.011^{*}$ & .051 & $.127^{*}$ & $.273^{* * *}$ & $-.239^{* * *}$ \\
202 & $.142^{* * *}$ & .058 & -.088 & $.148^{*}$ & .111 \\
203 & .014 & .030 & .055 & .106 & -.080 \\
204 & $.085^{*}$ & $.302^{* * *}$ & $.369^{* * *}$ & $.295^{* * *}$ & $.252^{* * *}$ \\
205 & $.175^{* * *}$ & $.080^{*}$ & $.152^{*}$ & .117 & -.007 \\
206 & $.289^{* * *}$ & $.263^{* * *}$ & $.488^{* * *}$ & $.327^{* * *}$ & .101 \\
207 & .066 & $.256^{* * *}$ & $.246^{* * *}$ & $.248^{* * *}$ & $.313^{* * *}$ \\
208 & $.161^{* * *}$ & .056 & $.376^{* * *}$ & $.128^{*}$ & $-.221^{* *}$ \\
209 & $.155^{* * *}$ & -.055 & -.037 & .058 & $-.197^{* *}$ \\
210 & .057 & .010 & .076 & -.064 & .003 \\
211 & $.193^{* * *}$ & $.295^{* * *}$ & $.385^{* * *}$ & $.194^{* *}$ & $.414^{* * *}$ \\
\hline
\end{tabular}

All of the participants' enumeration reports were more variable when they were tracking more objects. Also, the meanadjusted variation in enumeration reports was more directly correlated with the numerosity of the enumeration stimuli in the zero-tracking condition than in the four-tracking condition for 8 of the 11 participants. Furthermore, this correlation became decreasingly direct (i.e., $r$ decreased and/or became negative) across the zero-, two-, and four-tracking conditions for 4 of the 11 participants. While we cannot claim that all of the participants showed the same pattern of responses, the hallmark patterns of interference between the tracking and enumeration tasks that were seen in the averaged data (i.e., increased enumeration variability as the number of tracked items increased, particularly when enumerating small sets) were seen, at least in part, for almost all of the participants.

\section{Caveats and concerns}

Although our results indicate a direct trade-off between tracking and enumeration performance, neither Experiment 1 nor 2 addressed the possibility that the foreshortening of the subitizing range was not due to shared use of a specific visual mechanism, but rather to the delay between the presentation of the squares and the collection of the numerosity responses degrading the memory of the numerosity of the enumeration stimuli and/or to competition for general resources. We addressed this concern in Experiment 3 by including a dual-task in which participants had to enumerate while performing a task that, while comparably difficult to MOT, should not involve the use of visual indexes: a letter recall task. The timing structure of this enumeration and recall dual-task was matched to a tracking and enumeration dual-task run in the same session. Furthermore, neither Experiment 1 nor 2 included a baseline determination of the participants' tracking ability, but rather assumed a tracking limit of four or five items.
Thus, to ensure that our participants did indeed have typical baseline abilities, Experiment 3 included a condition to calculate each individual's tracking capacity, as well as his or her letter recall span.

\section{Experiment 3}

This experiment utilized two different dual-tasks in order to test whether the dual-task interference observed in Experiments 1 and 2 was due to a shared task-specific mechanism (i.e., an indexing mechanism) or only to effects of delay or shared general resources. In one block, participants enumerated while tracking (as in Experiments 1 and 2). In another block, participants enumerated while maintaining strings of three or five letters in working memory. In a third block, these tracking, enumeration, and recall tasks were completed individually to establish baseline performance. Importantly, the timing and visual characteristics of these single and dualtasks were identical, in order to ensure that these factors would not confound the results.

We determined that recall of three and five letters, respectively, was analogous in difficulty to tracking two and four discs. Studies have shown that adults can typically maintain six or seven items in verbal working memory (Miller, 1956). In the enumeration and recall dual-task, one of these slots would be needed to store the result of the enumeration assessment, leaving five or six slots free to use for the letter recall task. Previous studies (Pylyshyn, 1989; Pylyshyn \& Annan, 2006; Pylyshyn et al., 2008) indicated that people typically can track four or five items under speed and duration conditions similar to those used in the present study; this limit also was confirmed by pilot testing. Thus, recalling three or five letters is analogous to tracking two or four items in terms of capacity (i.e., two less than maximum capacity vs. at or near maximum capacity). We 
must note that Miller's estimate of a verbal working memory capacity of six or seven units is at the high end of the estimates in the literature. Chen and Cowan (2009), for example, estimated this span to be as low as three units. However, we believe it is more conservative to err on the side of a larger rather than a smaller verbal working memory span. Overestimating the span would exaggerate, rather than obscure, the effect of working memory load on numerosity evaluation, particularly between the low-load (three-letter) and no-load (zero-letter) conditions. Thus, this would not reduce our ability to determine whether the effects of MOT on subitizing are solely attributable to general cognitive load. Additionally, piloting indicated that the dual-task of enumerating while maintaining three or five letters in memory (respectively) was as difficult as the dualtask of enumerating while tracking two or four items.

\section{Method}

\section{Participants}

The participants were 37 undergraduate students (17 female) at the University of Notre Dame, participating for extra credit or to fill a course requirement.

\section{Apparatus}

The experiments were programmed in MATLAB R2010a using Psychophysics Toolbox version 3.0.8 (Brainard, 1997) and presented on Apple iMacs running Mac OS 10.5.8. The iMacs' screens had the same properties as those used in Experiments 1 and 2 . Responses were collected from a standard keyboard and mouse; participants used the keyboard to initiate each new trial at their own pace.

\section{Stimuli}

Tracking and enumeration stimuli The visual appearances of the tracking stimuli (white discs) and the enumeration stimuli (black squares) were identical to those seen on the iMac computers in Experiments 1 and 2. As in Experiment 2's shorter-duration conditions, speeds varied between 30 and 240 pixels (approximately $0.75^{\circ}-6^{\circ}$ ) per second, and squares were constrained to appear only in empty space. As in Experiment 1, four different total surface areas were used when constructing the squares for the enumeration stimuli: $30,000,40,000,50,000$, or 60,000 pixels.

Letter stimuli At the start of every trial in which the participants were asked to recall letters, the phrase "Remember these letters:" was presented at the center of the screen, followed by a set of three to eight randomly chosen lowercase consonants, excluding "y" (e.g., "Remember these letters: hsxqt"). These stimuli were presented in 28-point white Lucida Sans font.

\section{Order}

All six possible orderings of the single-task, tracking and enumeration dual-task, and enumeration and recall dualtask blocks were paired with each of the six possible orderings of the tracking, enumeration, and recall singletask trial sets within the single-task block. This yielded 36 unique orders, with each order assigned randomly to a single participant, with the exception of a single order that was used by 2 participants.

\section{General procedure}

Each participant completed one 290 -min session. The participant sat $\sim 60 \mathrm{~cm}$ from the test display; no restraints were used to restrict head or eye movements. When discs were present, a white fixation cross appeared in the center of the screen to encourage participants to remain focused on that location. No other controls for eye motion were used. The experimenter described the procedure in detail before the start of the experiment and reminded the participants of the instructions for each set of trials before they began a new task. A set of practice trials (three trials for the singletask trial set, and six for the dual-task trial sets) was administered at the start of each trial set to ensure understanding of the task.

Participants were told that they would perform three different kinds of tasks on a computer: a letter recall task, a disc-tracking task, and a square enumeration task. They were also told that sometimes they would be performing these tasks one at a time, and sometimes they would be asked to do two of the tasks simultaneously. They were further told that "all of the tasks look pretty much the same," but the particular task(s) they were asked to complete would change. Participants were instructed to concentrate on the task(s) at hand, to perform all tasks to the best of their ability, not to prioritize one task over another in the dual-task portions, and to answer as quickly and accurately as possible. After each trial was completed, the participants were prompted to press the space bar to initiate the next trial. Participants could choose to take a break at any point during the experiment by waiting to press the space bar.

Dual-task: Tracking and enumeration This task was like the tracking and enumeration dual-task used in Experiments 1 and 2, except that the zero-tracking trials were not included. The presentation timing was identical to that of the shorter- 
duration conditions in Experiment 2: 2,000 ms of identification, followed by $4,000 \mathrm{~ms}$ of motion, with the enumeration stimuli presented between 500 and 3,500 ms of motion. A total of 160 trials were administered to each participant in this block, with 40 trials in each of the tracking/report conditions (4 trials for each numerosity). Each of the four possible surface areas described above was used once for each square numerosity (one to nine squares) for each of the four tracking/report conditions, with an additional 4 trials in which zero squares were presented.

Dual-task: Enumeration and recall This task was like the tracking and enumeration dual-task, except that a 2,000-ms presentation of to-be-recalled letters replaced the 2,000-ms flashing of to-be-tracked discs. Each trial began with an initial gray screen indicating a string of three or five random consonants that the participant needed to remember. After 2,000 ms (60 frames), this screen was replaced by eight discs, which moved randomly around the screen for 4,000 ms (120 frames). At a random point between $500 \mathrm{~ms}$ (15 frames) and $3,500 \mathrm{~ms}$ (105 frames) of movement, the enumeration stimulus was flashed for $33.3 \mathrm{~ms}$ ( 1 frame). The discs disappeared after the remainder of the 4,000-ms movement duration. Next, in a random $50 \%$ of the recall-three and recallfive trials, the participant was prompted to type in the to-berecalled letters by the words "Please type the letters you saw, in order, CAREFULLY!" appearing in the middle of the screen. Participants typed in their answers via the keyboard, pressing the return key to submit their answer. Only letter sets that contained the correct number of letters could be successfully entered. Input letters were presented on the screen to the right of the prompt. Prior to entering the answer, each participant could correct the letter string by using the delete key to remove the final letter in the current string. This recall report was skipped on the other random $50 \%$ of trials. Next, the participants were prompted to use the keyboard to indicate the number of squares that had appeared during the trial. Each participant completed 160 trials in this block, with 40 trials in each of the following conditions: recall-three with recall report, recall-three without recall report, recall-five with recall report, and recall-five without recall report (4 trials per numerosity). Each of the four possible surface areas described above was used once for each square numerosity (one to nine squares) for each of the four recall/report conditions, with an additional 4 trials in which zero squares were presented.

Single-task: Enumeration This task was much like the zero-tracking trials used in Experiments 1 and 2. Each trial began with an initial gray screen with eight identical discs in a random configuration. These remained still for $2,000 \mathrm{~ms}$ (no targets were identified). The discs began moving randomly around the screen for 4,000 ms (120 frames). At a random point between $500 \mathrm{~ms}$ (15 frames) and 3,500 ms (105 frames) of movement, the enumeration stimulus appeared for $33.3 \mathrm{~ms}$ ( 1 frame). At the end of the 4,000-ms movement duration, the discs disappeared and the participant was prompted to use the keyboard to indicate the number of squares that had appeared during the trial. In this block, a total of 40 trials were administered to each participant, 4 for each numerosity. Each of the four possible surface areas described above was used once for each numerosity (one to nine squares), with an additional 4 trials in which zero squares were presented.

Single-task: Object tracking There were two sets of trials in this block, one set in which participants tracked 2-4 of 8 discs (small set), and a second in which participants tracked 4-6 of 12 discs (large set). For the small set, each trial began with an initial gray screen with eight identical discs in a random configuration. At this point, 2-4 of these discs were identified as the to-be-tracked targets by flashing blue ten times over 2,000 ms (60 frames, 6 frames per flash). After the flashing ceased, the objects began moving randomly around the screen for 4,000 ms (120 frames). At a random point between $500 \mathrm{~ms}$ (15 frames) and 3,500 ms (105 frames) of movement, the background turned to a lighter gray color and three, six, or nine squares (total area 30,000 or 60,000 pixels) appeared for $33.3 \mathrm{~ms}$ (1 frame). Then the screen background color reverted to the original gray, and the discs continued moving for the remainder of the 4,000-ms duration. After the discs stopped moving, the participant was prompted to select the tracked targets using the mouse. The large set followed the small set and proceeded similarly, except that there were 12 total discs, 4-6 of which were flashed as targets. In this block, a total of 36 trials were administered to each participant, with 6 trials for each tracking/set-size pair.

Single-task: Letter recall These trials were like the singletask object-tracking trials, with two exceptions. (1) Instead of the 2,000-ms period of target identification, each trial began with an initial gray screen indicating a string of three to eight random consonants that the participant needed to remember. This was followed by a 4,000-ms period of disc motion, including a brief presentation of squares, like that used in the single-task object-tracking trials. (2) After this period of movement, the discs disappeared and the participant was prompted to type in the to-be-recalled letters. In this block, a total of 36 trials were administered to each participant, with 6 trials in each of the three- to eight-letter recall conditions.

Results and discussion

The results of this experiment again supported our hypothesis that the subitizing of visual items and MOT 
share a common visual mechanism. The discussion of results is divided into eight parts: (1) We establish that the baseline tracking and recall abilities were normal. (2) We establish that participants were as accurate at recalling three and five letters in the enumeration and recall dual-task as they were at tracking two and four items in the tracking and enumeration dual-task, further supporting our hypothesis that these tasks were equally difficult. (3) We show that in the tracking and enumeration dual-task, tracking performance was high in all enumeration conditions. (4) We show that in the enumeration and recall dual-task, recall performance was high in all enumeration conditions. (5) We establish that participants enumerated successfully in all single- and dual-task enumeration conditions. (6) We show that the direct-assessment hypothesis was not supported. (7) We show that, in accordance with our secondary-mechanism hypothesis, the subitizing range decreased as tracking load increased. (8) We show that a different interference pattern was seen in the enumeration and recall dual-task than in the tracking and enumeration dual-task, indicating that the interference seen between tracking and enumeration was not merely the result of general cognitive load or of the delay between the presentation of enumeration stimuli and the enumeration response. Note that we only included in our analyses and graphs enumeration reports from trials in which neither tracking reports nor recall reports were collected.

\section{Baseline tracking and recall abilities were normal}

In order to check that the participants had typical baseline abilities on the tracking and recall tasks, these baselines were estimated on the basis of individuals' performance in the tracking and recall single-tasks. Performance was found to be within the normal range.

Tracking limits Tracking performance was high overall, as is illustrated in Fig. 11. Individual participants' tracking limits were estimated as the highest tracking level at which the proportion of trials a participant tracked perfectly was significantly above chance for that level and for all lower tracking levels. For example, a person able to track only 3 items would have only a $20 \%$ chance of performing a 4 out-of- 8 tracking trial perfectly, since he or she would have to guess which of the 5 items that were not tracked was the remaining target. Such a person would have less than a $5 \%$ chance of performing perfectly on at least three out of the six trials of this type (see Hulleman, 2005, for a discussion of these calculations). Thus, if a person performed perfectly on at least three out of six such trials, he or she was above chance, and the tracking limit was determined to be at least 4 , provided that this individual was also above chance on 2out-of- 8 and 3-out-of- 8 tracking (which all participants were). The mean tracking limit was found to be 4.57 discs
$(S E=0.113)$. The distribution of tracking limits is displayed in Fig. 11. This is in line with limits found under these speed and duration conditions in other studies (Alvarez \& Franconeri, 2007; Pylyshyn \& Annan, 2006).

Letter recall spans Letter recall performance was high overall. The rates of perfect recall performance-the proportion of trials on which all letters were recalled correctly and in their correct locations - are displayed in Fig. 11. Individual participants' recall limits were estimated based on the highest recall level for which the proportion of trials a participant recalled perfectly was significantly above chance for that level and for all lower recall levels. For example, if a person were able to recall 4 items, he or she would have only a $5 \%$ chance of performing a 5 -letter trial perfectly, since he or she would have to guess the fifth consonant, which was not recalled. Such a person would have less than a $5 \%$ chance of performing perfectly on at least two out of the six trials of this type. Thus, if a person performed perfectly on at least two out of six such trials, that person was considered to be above chance, and the letter span was determined to be at least 5 , provided that he or she was also above chance on the 3- and 4-letter recall conditions (all participants were). The mean letter span was found to be 6.73 $(S E=.148)$. The distribution of recall limits is displayed in Fig. 11. These results are in line with letter spans found in prior research (e.g., Miller, 1956).

\section{Performance}

Participants were equally accurate at tracking and letter recall in the dual-tasks Overall, tracking performance in the tracking and enumeration dual-task was high, with rates of perfect performance averaging $91 \%(S E=1.2 \%)$ in the two-tracking condition (chance of guessing all items = $3.6 \%)$ and $77 \%(S E=2.2 \%)$ in the four-tracking condition (chance of guessing all items $=1.4 \%$ ). Each individual participant performed significantly above chance in both conditions, with rates of perfect performance ranging from $70 \%$ to $100 \%$ in the two-tracking condition and from $53 \%$ to $98 \%$ in the four-tracking condition. Letter recall performance in the enumeration and recall dual-task was also quite high, with rates of perfect performance averaging at $89 \%(S E=1.6 \%)$ in the recall-three condition (chance of guessing all letters correctly $<0.1 \%)$ and $73 \%(S E=$ $3.0 \%$ ) in the recall-five condition (chance of guessing all letters correctly $<0.0001 \%$ ). Each individual participant performed significantly above chance in both conditions, with perfect scores ranging from $55 \%$ to $100 \%$ in the recallthree condition and from $13 \%$ to $98 \%$ in the recall-five condition. The rate of perfect performance on the recallthree task did not differ significantly from the rate of perfect performance on the two-tracking task, nor did the rate of 

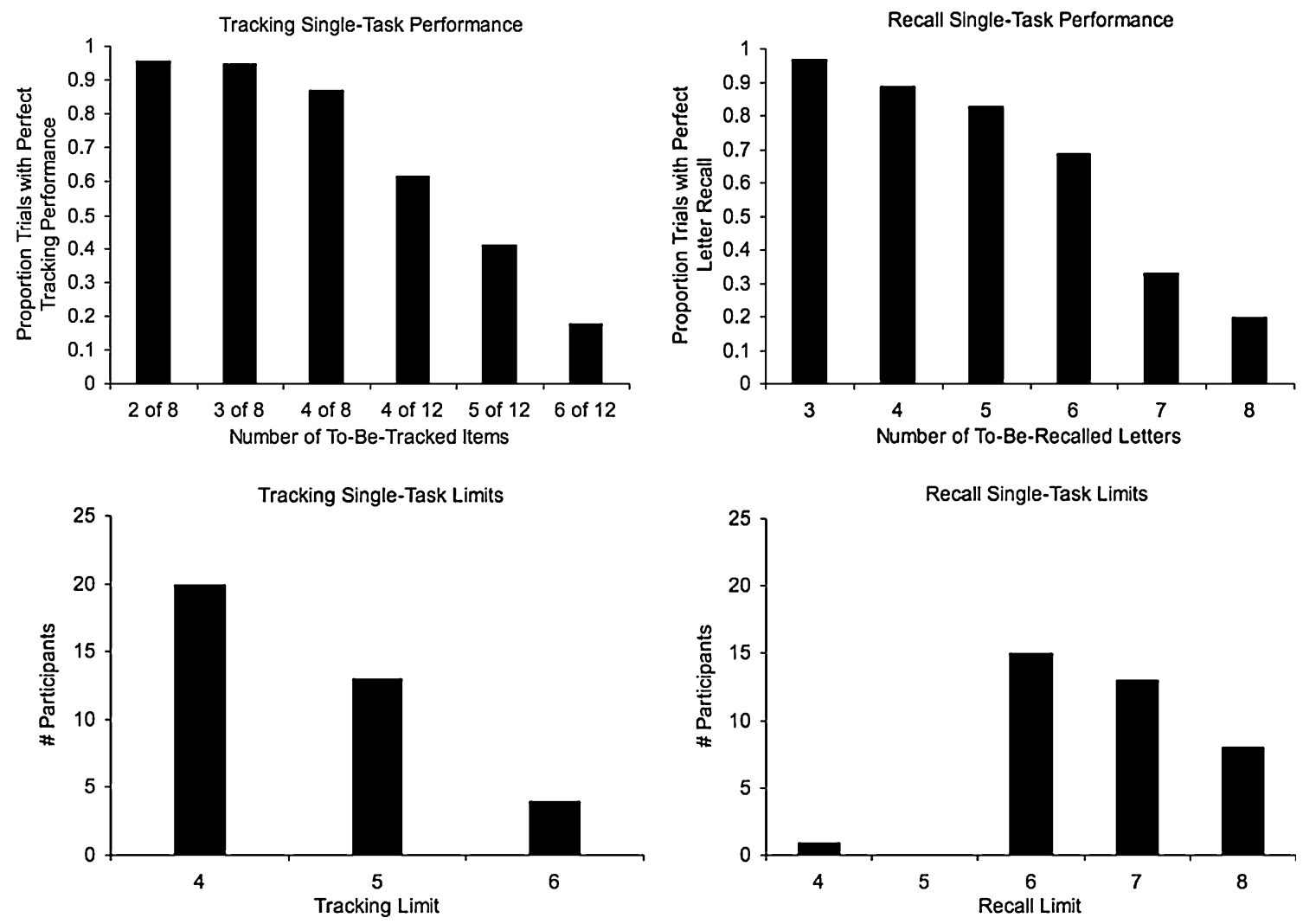

Fig. 11 Experiment 3: Tracking and recall single-task results. Proportions of trials with perfect tracking performance in the tracking single-task (top left). Proportions of trials with perfect recall performance in the recall

perfect performance on the recall-five task differ significantly from the rate of perfect performance on the fourtracking task. This confirms that the two tasks were approximately equal in overall difficulty.

Tracking in the tracking and enumeration dual-task was near maximum for all numerosities While there was some decrement in the proportion of perfectly tracked trials in the four-tracking condition as the numerosity of the enumeration stimulus increased, the slope of the effect size was very small (slope $=-.011, S E=.004, t=-2.9, p<.005, R^{2}=$ .006 ; see Fig. 12). Therefore, we concluded that tracking performance was at or near maximum throughout the numerosity conditions, and thus any dual-task trade-offs should be seen in the subitizing range.

Recall in the enumeration and recall dual-task was near maximum for all numerosities While there was some decrement in the proportion of perfectly recalled trials in the recall-three condition as the numerosity of the enumeration stimulus increased, the effect size was very small (slope $=-.011, S E=.003, t=-3.8, p<.001, R^{2}=.010$; see Fig. 12). Therefore, we concluded that recall performance was at or near maximum throughout the numerosity

single-task (top right). Numbers of participants with various estimated tracking limits (bottom left). Numbers of participants with various estimated recall limits (bottom right)

conditions, and thus any dual-task trade-offs should be seen in the subitizing range.

Participants enumerated successfully in the single-task and in both dual-tasks Enumeration performance was high in the enumeration portion of the single-task block. A linear regression found a strong positive correlation between the number of squares presented and the enumeration responses (slope $=.873, S E=.007, t=7.9, p<.001, R^{2}=.909$ ), with a constant of .301 $(S E=.038)$ (enumeration response $=.873 *$ number of squares +.301 ; see Fig. 13). Enumeration performance during the tracking and enumeration dual-task was also high (see Figs. 13 and 14). A linear regression found a strong positive correlation between the number of squares presented and the enumeration responses $(t=95.1, p<.001$, $\left.R^{2}=.801\right)$. The best-fit line had a slope of $.754(S E=.008)$, with a constant of $.747(S E=.042)($ enumeration response $=$ $.754 *$ number of squares +.747 ; see Fig. 13). Participants were also able to enumerate effectively during the enumeration and recall dual-task (see Figs. 13 and 14). A linear regression found a strong positive correlation between the number of squares presented and the enumeration responses ( $\left.t=125.9, p<.001, R^{2}=.843\right)$. The best-fit line had a slope of $.829(S E=.007)$, with a constant of $.489(S E=.035)$ 
Fig. 12 Experiment 3: Proportions of trials with perfect tracking reports in the tracking and enumeration dual-task (left) and perfect recall reports in the enumeration and recall dual-task (right) as a function of tracking or recall condition and the number of squares displayed. Error bars represent standard errors

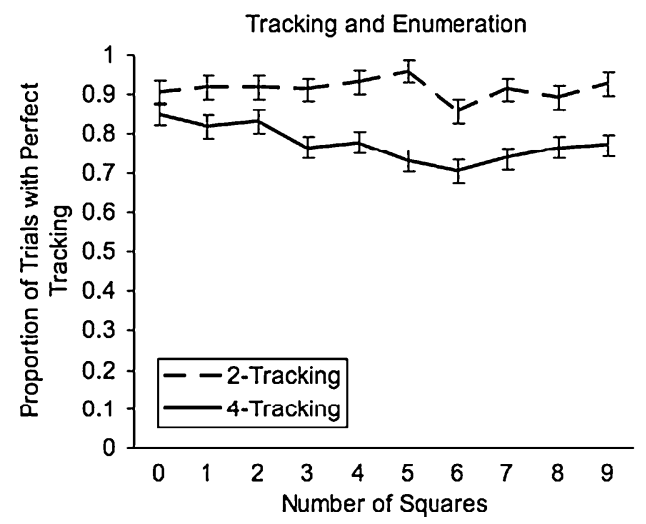

(enumeration response $=.829 *$ number of squares +.489 ; see Fig. 13).

\section{Testing the direct-assessment hypothesis}

When analyzing trials in which only one to four squares were presented (the typical subitizing range), a direct correlation was found between the number of items tracked and the participants' numerical responses $(r=.085, p=$ .003). A linear regression on these data found that numerical responses increased on average by 0.059 ( $S E=$ $0.019, t=3.1, p<.01$; intercept $=2.488, S E=0.049, t=$
50.4, $p<.001, R^{2}=.073$ ) for every item tracked. This correlation was also significant for 2 of the 37 individual participants $(r=.325, p=.024 ; r=.391, p<.01)$. For the participants with a significant correlation, the coefficients were $0.359(S E=0.125, t=2.9, p<.01$; intercept $=2.344$, $\left.S E=0.302, t=8.0, p<.001, R^{2}=.153\right)$ and $0.297(S E=$ $0.127, t=2.3, p=.024$; intercept $=2.385, S E=0.329, t=$ $\left.7.3, p<.001, R^{2}=.105\right)$. These slopes and effect sizes are quite small, and as such do not support the conclusion that cardinalities subitized from small sets increased in one-toone correspondence with the number of items tracked. Also, participants again showed some subitizing ability
Fig. 13 Experiment 3:

Mean enumeration responses in the enumeration single-task (top), tracking and enumeration dual-task (bottom left), and enumeration and recall dualtask (bottom right) as a function of tracking or recall condition and the number of squares displayed. Error bars represent standard deviations. The gray dash-dotted lines indicate the correct numerosity
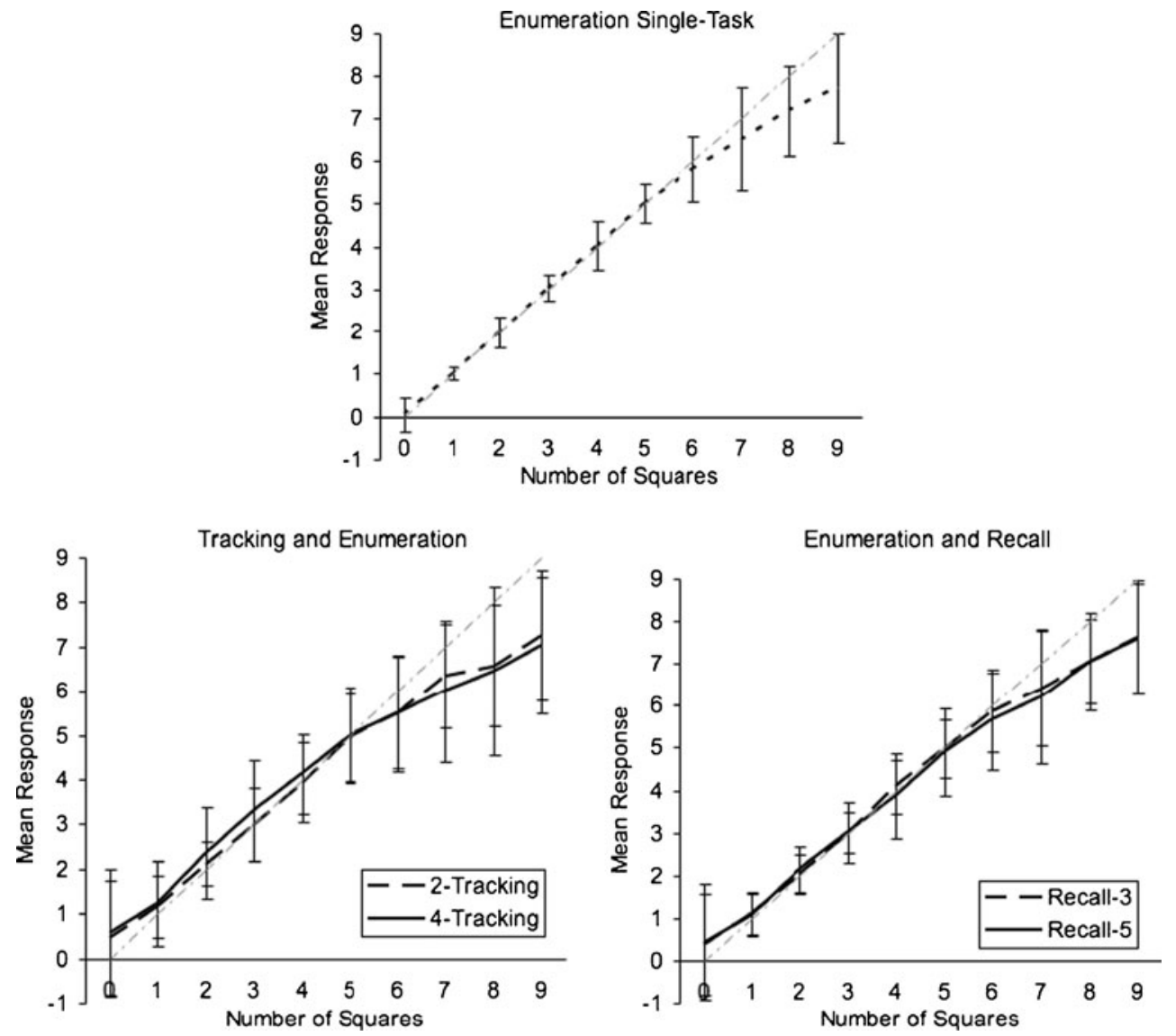
Fig. 14 Experiment 3: Proportions of trials with correct enumeration responses in the enumeration single-task (top), tracking and enumeration dualtask (bottom left), and enumeration and recall dual-task (bottom right) as a function of tracking or recall condition and the number of squares displayed. Error bars represent standard errors
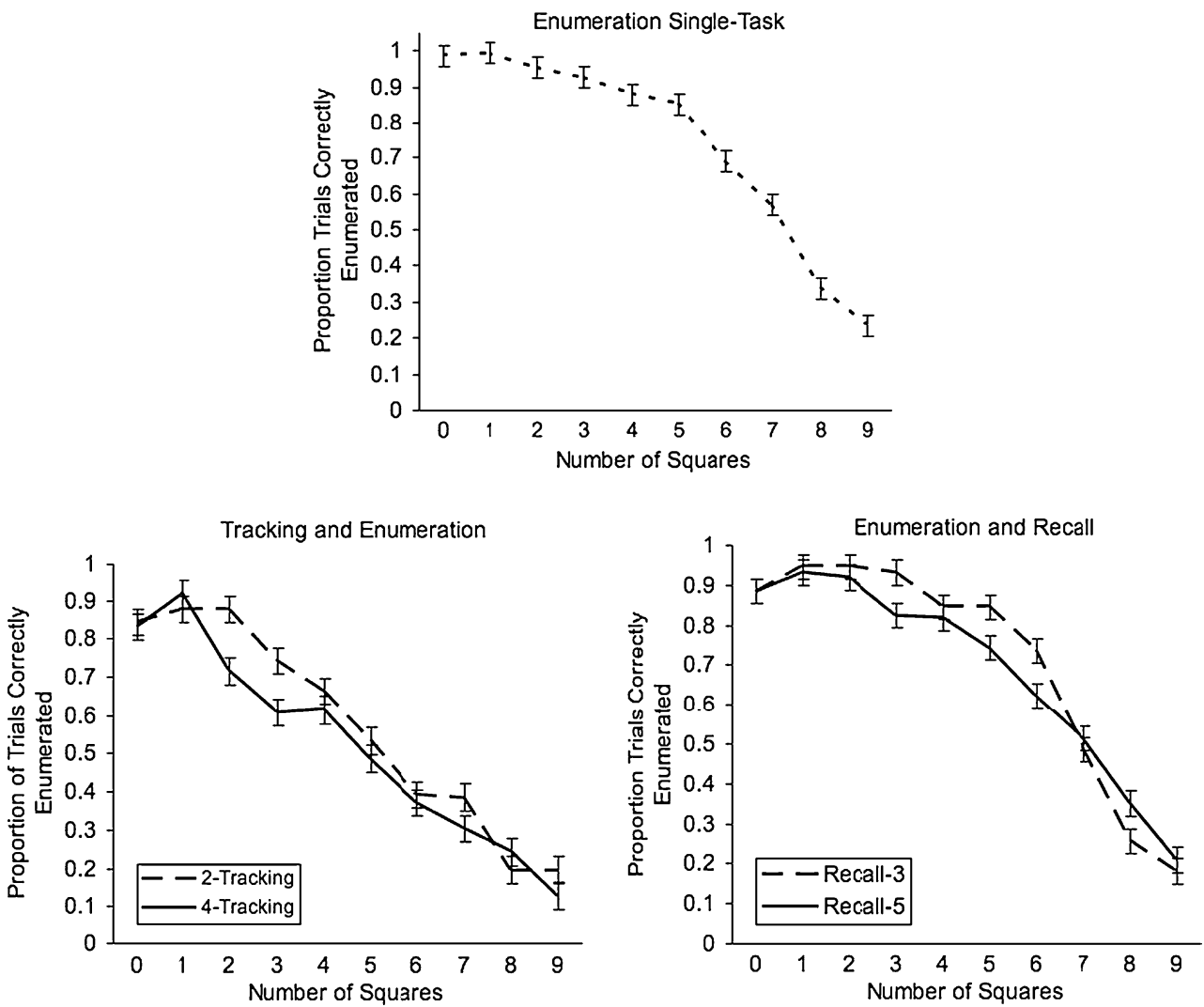

when tracking two items, offering evidence against the alternative hypothesis that people subitize via direct assessment, but only when all of their indexes are available.

\section{Testing the secondary-mechanism hypothesis}

The interference of tracking on enumeration was quite similar to that seen in Experiments 1 and 2. This was confirmed by a 3 (tracking condition: zero, two, or four items) $\times 6$ (enumeration condition: two to seven squares presented) repeated measures ANOVA run on the CVs of the participants' enumeration responses. Data from the enumeration single-task were treated as the zero-tracking condition. There was a significant main effect of tracking condition $\left[F(2,72)=32.9, p<.001, \eta_{p}^{2}=.478\right]$, but not of enumeration condition. However, the interaction between these two factors was significant $[F(10,360)=2.8, p<.01$, $\left.\eta_{p}^{2}=.073\right]$ : When participants were tracking more items, their enumerations of small numerosities were more variable. These variability levels tended to converge as the numerosities of the enumeration stimuli increased (see Fig. 15). These results again indicate that MOT and subitizing share common resources.

Furthermore, the subitizing range decreased as the number of objects tracked increased. As in Experiments 1 and 2, we estimated the subitizing range via the divergence and convergence of the CVs of the various tracking conditions. The subitizing ranges were estimated to be 6 in the zero-tracking condition, 2.5 in the two-tracking condition, and 0.5 in the four-tracking condition (see Fig. 15). Confirming these estimates of the subitizing range, visible drops in correct enumeration rates can be observed at similar values in Fig. 14. In the enumeration single-task (zero-tracking), there was a sharp decline in the rate of correct responding that seems to indicate a subitizing range of about 5. An abrupt decline in the two-tracking condition seems to indicate a subitizing range of about 2 . In the four-tracking condition, this decline seems to indicate a subitizing range of about 0 or 1 . As before, a continuous trade-off between subitizing range and tracking load was seen. These findings add further support to the hypothesis that subitizing and MOT share a common visual mechanism.

Recall interfered with subitizing differently than did MOT The pattern of interference seen in the enumeration and recall dualtask was different from that seen in the tracking and enumeration dual-task. We investigate this interference pattern via a 3 (recall condition: zero, three, or five letters) $\times 6$ (enumeration condition: two to seven squares presented) repeated measures ANOVA examining the $\mathrm{CVs}$ of the participants' enumeration responses. Data from the enumeration single-task were treated as the recall-zero condition. Both enumeration condition $\left[F(2,72)=10.5, p<.001, \eta_{p}^{2}=\right.$ $.226]$ and recall condition $\left[F(5,180)=3.4, p<.01, \eta_{p}^{2}=\right.$ 


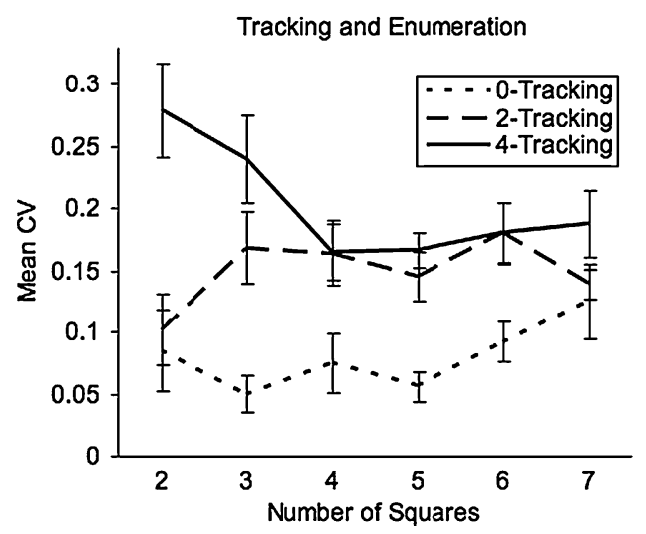

Fig. 15 Experiment 3: Mean coefficients of variation $(\mathrm{CVs})$ of enumeration from (left) the enumeration single-task (0-tracking) and the tracking and enumeration dual-task (2-tracking and 4-tracking), and from

.086] had significant main effects, but the interaction was not significant $\left[F(10,360)=0.7, p=.769, \eta_{p}^{2}=.018\right]($ see Fig. 15). Enumeration CVs of the recall-three and recall-zero conditions did not diverge; however, an increase in CVs was seen in the recall-five condition.

We used the method of divergence and convergence of $\mathrm{CVs}$ to estimate the subitizing range in the recall-three and recall-five conditions, as we had done for the dual-task with tracking. As discussed above, the recall-zero condition (which also served as the zero-tracking condition) was estimated to yield a subitizing range of 6 . Since the CVs in recall-zero and recall-three conditions never diverged, the recall-three condition could also be estimated as yielding a subitizing range of 6 . We estimated the subitizing range in the recall-five condition to be 2 , the last numerosity before which the CVs in the recall-five condition diverged from the CVs of the recall-zero and recall-three conditions. Confirming these estimates of the subitizing range, visible drops in correct enumeration rates can be observed at similar numerosities in Fig. 14. As in the recall-zero condition, a sharp decline in the rate of correct enumeration reports for the recall-three condition suggests a subitizing range of about 5. In the recall-five condition, the precise location of this decline is less clear, but it occurs somewhere in the range of 2-4. While it does appear that heavily loading working memory can impair subitizing, a continuous trade-off between subitizing and working memory load was not seen.

These results further support our hypothesis that the interference between subitizing and MOT is due to a shared mechanism rather than solely to competition for general resources and/or to the delay between the presentation of enumeration stimuli and the collection of enumeration responses. If the interference patterns were due to greater memory degradation for the numerosity assessment during the delay between presentation and response as task load increased, one would expect to find no difference between

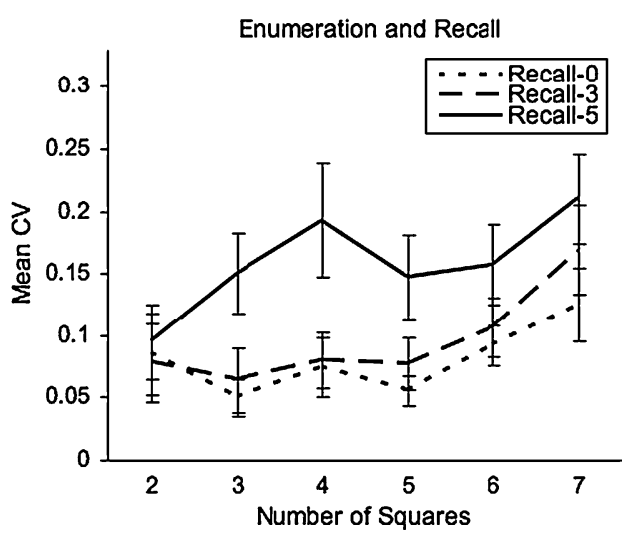

(right) the enumeration single-task (recall-0) and the enumeration and recall dual-task (recall-3 and recall-5) as a function of tracking or recall condition and the number of squares displayed. Error bars represent standard errors

the recall and MOT dual-tasks. Alternatively, one could anticipate a greater effect of letter load than of MOT load on enumeration, as, presumably, a verbal working memory task would be more likely to interfere with the verbal working memory of a numerosity assessment than would a visual tracking task. Likewise, if the interference pattern were due to competition for general resources, similar interference patterns should have been observed in the two dual-tasks, since the general load was approximately equal in these tasks. Contrary to these predictions, no differences were seen between the CVs for enumeration performance in the recall-three and recall-zero conditions. Furthermore, although a reduction in the subitizing range was seen for the recall-five condition, subitizing was still present for numerosities up through 2 . This partial reduction in the subitizing range when recalling five letters is predicted if subitizing and MOT shared a common mechanism. Previous findings from dual-task studies (e.g., Allen et al., 2006) have indicated that when MOT tasks are performed with nonvisual tasks that load working memory, participants were typically able to track only three rather than four or five objects. Thus, the reduction seen in the subitizing range when under a heavy working memory load was similar to the reduction previously seen in tracking limits under heavy working memory load.

\section{Meta-analysis of Experiments 1, 2, and 3}

With three dual-task experiments completed, sufficient data points had been collected to analyze the relationship between subitizing range and tracking load. A linear regression of the subitizing estimates from Experiments 1, 2, and 3 found a strong negative correlation between the subitizing ranges and the number of items tracked $\left(t=-6.1, p<.005, R^{2}=.840\right)$. Furthermore, the best-fit line was found to have a slope of $-1.04(S E=0.172)$ with a constant of $4.58(S E=0.444$, $t=10.3, p<.001$ ) (subitizing range $=-1.04 *$ items tracked +4.58 ; see Fig. 16 ). These are precisely the results 
one would expect if subitizing utilized the same mechanism that supports MOT: Participants in these experiments could typically subitize up to four or five items, but that amount decreased by one for every item tracked.

\section{General discussion}

Subitizing is not achieved by directly assessing the "fullness" of one's indexes

As we previously discussed, one possible account of the subitizing effect is that people are able to use the indexing system to directly assess small set numerosities: An index is attached to each to-be-enumerated item, and the "fullness" of the indexing system is used to determine exactly how many items are present. If this were the case, one would predict that, particularly in the small number range, people would overestimate small set numerosities when their indexes were engaged with items that were not members of the to-be-enumerated set. If, for example, a person was tracking two items and needed to simultaneously enumerate a set composed of two additional items, four indexes would be in use, and a direct reading of the "fullness" of the indexing system would result in the erroneous response of "four." Thus, one would expect that sets would be overestimated by one for every index concurrently engaged in another task, such as tracking. No such effect was seen in our experiments. Although in Experiments 2 and 3 there was a small but significant increase in the cardinality of the participants' small-set enumerations as the number of to-be-tracked items increased (and, consequently, the number of occupied indexes), responses increased by significantly less than 0.1 for each additional object tracked. This is much less than the slope of 1 that would be expected if participants had been determining set

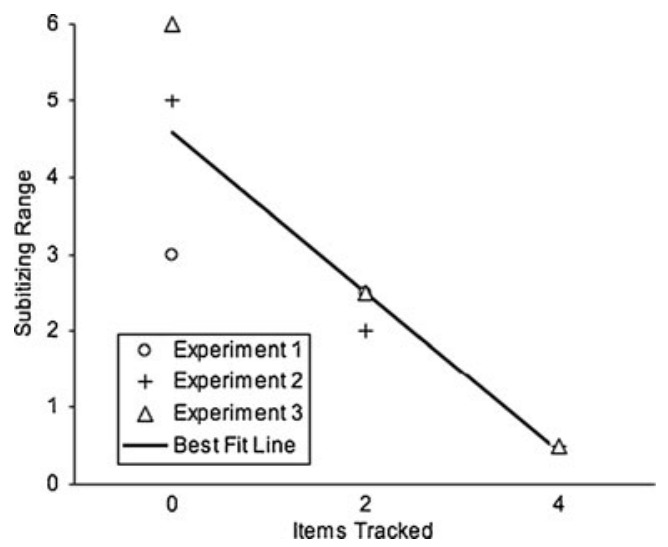

Fig. 16 Estimated subitizing ranges in Experiments 1, 2, and 3 as a function of the number of items tracked cardinality on the basis of the "fullness" of their indexing mechanism. The small effect that was found could be explained by participants occasionally including one or more of the items that they tracked in the to-beenumerated set. Alternatively, if people did subitize by judging index "fullness," but only when all indexes were available for the task, we would not expect to see any subitizing while any moving items were being tracked. Contrary to this prediction, participants showed some subitizing ability when tracking two items. It seems clear that participants were not relying on a direct assessment of index activity to enumerate small sets.

Tracking and subitizing share a common visual mechanism

Although our results do not support the direct-assessment hypothesis of subitizing, the secondary-mechanism hypothesis is supported. The results indicate that tracking and subitizing share specific resources. Participants' small-set enumerations were significantly more variable when they tracked more objects; this difference among tracking levels diminished as the size of the to-be-enumerated set increased. Additionally, in each experiment our estimates of the subitizing range decreased as the number of items tracked increased, and indeed, a meta-analysis of these estimates showed that the size of the subitizing range decreased by 1 for every item tracked. A different interference pattern was seen between subitizing and letter recall: No decrease in the subitizing range was observed when recalling three letters (see Fig. 15), and only a partial reduction was seen when participants were recalling five letters. This reduction paralleled the partial reduction in tracking ability seen in past studies when people tracked under a heavy working memory load (Allen et al., 2006). Given the differences in the interference patterns observed between the enumeration and letter-recall dual-task and the tracking and enumeration dual-task, it is unlikely that the performance trade-off observed between subitizing and MOT in Experiments 1, 2, and 3 is entirely attributable to competition for general resources. Instead, these results indicate that the interference between subitizing and tracking is due, at least in part, to competition for specific visual resources, namely the visual indexing mechanism.

\section{Caveats and concerns}

As a caveat to this conclusion, we must note that both visual working memory and attention are closely associated with visual indexing in early vision. For example, visual working memory may help keep a record of which indexes are involved in tracking (Pylyshyn \& Storm, 1988) or may encode the locations of tracked objects when they temporarily disappear (Horowitz, Birnkrant, Fencsik, Tran, \& 
Wolfe, 2006). Likewise, attention may be needed to maintain performance when scene characteristics change, and thereby increase tracking difficulty (Tombu \& Seiffert, 2008). Additionally, modality-specific interference has been found between visual working memory tasks and both MOT (Fougnie \& Marois, 2006) and subitizing (Piazza, Fumarola, Chinello, \& Melcher, 2011) performance, which can be attributed to the individuation stage of visual processing. Such studies demonstrate that intricate links exist between these visual systems and indicate a constant flow of information between them under normal conditions. Consequently, our results do not rule out the possibility that the interference observed between tracking and subitizing is due to competiton for visual working memory or visuospatial attention, rather than specifically to competition for visual indexes (see Egeth, Leonard, \& Palomares, 2008; Olivers \& Watson, 2008; Vetter et al., 2008). Therefore, further studies are warranted in order to determine whether vision-specific interference is due to the common use of visual indexes, rather than to competition for closely related visual resourses in early vision.

Furthermore, it is important to note that the presence of vision-specific interference does not preclude the possibility that competition for general resources may also partially contribute to the interference pattern seen between subitizing and MOT. Indeed, considering our finding that a heavy working memory load decreased the subitizing range, as well as previous findings that a heavy working memory load decreases the tracking limit (Allen et al., 2006), it would be more surprising if competition for general resources did not play some role in the interference of tracking on subitizing. Further studies will be needed to determine exactly what portion of this interference is attributable to competition for general resources.

The approximate number system might

be used to enumerate indexes

It seems that indexes may indeed be responsible for the subitizing effect, but not via a direct association of the "fullness" of the indexing system to a cardinality label. Rather, the numerosity of indexes assigned to the to-beenumerated set must be assessed by a secondary system, as in Trick and Pylyshyn's (1993, 1994b) account. But what system? Given the speed with which subitizing responses can be produced and the fact that subitizing occurred when counting was prevented by short stimulus durations, it is unlikely that verbal counting was used to enumerate these indexes. We also find it unparsimonious to posit that a separate enumeration system exists solely for the purpose of enumerating subsets of visual indexes. Rather, one account of our results is that indexes aid ANS enumeration: The subitizing effect may be a result of the indexing mechanism effectively removing variability due to misses and multiple entries of items into the ANS system.

In order for the numerosity of a set to be evaluated by the ANS, all members of that set must be entered into the system exactly once. In the accumulator model (Meck \& Church, 1983), the ANS is conceived of as a graduated cylinder, to which one "cup" is added for every enumerated item. The value of the set can be estimated by reading the "height" of this accumulator (Cordes et al., 2001). When items are entered more than once, or not at all, the ANS will produce inaccurate estimates, not because of the variability in its numerosity representation, but rather due to the fact it was representing the wrong numerosity. To illustrate, consider a set of five items. If all five items were entered correctly into the accumulator, the accumulator would represent the set's quantity with scalar variability around five. However, if only four of the five items in the set were entered into the accumulator, because one of the items was "missed," the accumulator would instead represent the set's quantity with scalar variability around four. One could expect there to be some error in the enumerations produced due to the to-be-enumerated items being entered into the system multiple times or not at all (C. Gallistel, personal communication, December, 2008). By the law of large numbers, one could expect this noise to be reduced to a proportional bias for large sets, but in the small number range, it would result in an increase in the $\mathrm{CV}$ greater than that inherent to the accumulator. Indexes could effectively eliminate this source of variability in the small number range by keeping track of which items have been accumulated, thus preventing misses and multiple entries. When there are insufficient indexes available to keep track of which items have been entered, the $\mathrm{CV}$ of the estimates would increase due to these entry errors. This would result in the rise in the CVs of enumeration reports for numerosities beyond the subitizing range.

This would also explain why studies using different reporting methods, such as reporting numerosity indirectly (i.e., by clicking on a screen to indicate the locations of briefly presented items) show very low error rates for as many as six items, but when cardinal symbols are used to report numerosities, there is an increase in error rates for set sizes greater than 4 (Haladjian \& Pylyshyn, 2011). Accurately representing a collection of items does not necessarily entail accurately representing the numerosity of that collection in an immediately accessible fashion (Gallistel, 2007). That is, it may be possible to mentally represent a collection of items without explicitly knowing how many items are represented, necessitating the use of a secondary mechanism or other heuristic to determine the numerosity of the set that contains the members of that collection. If the 
number of items one can simultaneously represent exceeds the numerosity that this secondary system can assess with little or no variability, as would be the case if a set of six mentally represented items were being assessed by an accumulator with an effective subitizing range of 4 (see Fig. 1), one would be able to accurately report fewer items than one could represent mentally.

Two caveats to this hypothesis are that (1) the error due to the variance in the representation of numerical magnitudes by the ANS would still be present, and (2) previous estimates (Cordes et al., 2001) have placed the CV of the ANS at about .15, which, as previously discussed, would yield a subitizing range of 1 or 2 , not 4 or 5 . However, the acceleration in the error rates and/or the increase in reaction times after the small number range - the hallmark pattern of the subitizing effect-would still be seen when the size of the to-be-enumerated set exceeded the number of available indexes, because the variability due to entry error would add to the inherent variability of the ANS for estimates of all subsequent values. Additional studies will be needed to test the viability of this hypothesis.

\section{Conclusions}

Our results suggest that the subitizing of visual sets and MOT do not merely share general resources, such as working memory, but in fact utilize the same underlying visual mechanism. The subitizing range decreased directly with the number of items that participants tracked, with a one-to-one trade-off. No such relationship was observed in a dual-task experiment that required enumeration to be performed concurrently with a working memory task (i.e., letter recall) of difficulty similar to that of the tracking task. Additionally, subitizing was found not to have been accomplished by directly associating an amount of active indexes to a numerosity stored in memory. Additional studies will be needed to determine the exact method by which a subset of assigned visual indexes might be enumerated to produce the subitizing effect (e.g., by reducing variability of ANS estimates). Also, the relationship between visual indexing and other visual mechanisms (such as visuospatial attention) needs to be further examined in order to better understand the cause of the performance trade-offs observed in the present study and the stage in early visual processing at which they occur.

Author note This research was supported by Rochel Gelman's Rutgers University institutional research funds (D.L.C.) and Grant NSF IGERT DGE 0549115 (H.H.H.). The authors thank Randy Gallistel, Rochel Gelman, Percival Mathews, Nicole McNeil, and Zenon Pylyshyn for their support and helpful comments on drafts of the manuscript. D.L.C. was at the Department of Psychology at Rutgers University, New Brunswick, and is now at the Department of Psychology at the University of Notre Dame.

\section{References}

Allen, R., McGeorge, P., Pearson, D. G., \& Milne, A. (2006). Multiple-target tracking: A role for working memory? Quarterly Journal of Experimental Psychology, 59, 1101-1116.

Alvarez, G. A., \& Franconeri, S. L. (2007). How many objects can you track? Evidence for a resource-limited attentive tracking mechanism. Journal of Vision, 7(13), 14:1-10. doi:10.1167/7.13.14

Alvarez, G. A., Horowitz, T. S., Arsenio, H. C., Dimase, J. S., \& Wolfe, J. M. (2005). Do multielement visual tracking and visual search draw continuously on the same visual attention resources? Journal of Experimental Psychology: Human Perception and Performance, 31, 643-667.

Atkinson, J., Campbell, F. W., \& Francis, M. R. (1976). The magic number $4 \pm 0$ : A new look at visual numerosity judgements. Perception, 5, 327-334. doi:10.1068/p050327

Brainard, D. H. (1997). The Psychophysics Toolbox. Spatial Vision, 10, 433-436. doi:10.1163/156856897X00357

Burr, D. C., Turi, M., \& Anobile, G. (2010). Subitizing but not estimation of numerosity requires attentional resources. Journal of Vision, 10(6), 20:1-10. doi:10.1167/10.6.20

Camos, V., \& Tillmann, B. (2008). Discontinuity in the enumeration of sequentially presented auditory and visual stimuli. Cognition, 107, 1135-1143.

Cavanagh, P., \& Alvarez, G. A. (2005). Tracking multiple targets with multifocal attention. Trends in Cognitive Sciences, 9, 349-354. doi:10.1016/j.tics.2005.05.009

Chen, Z., \& Cowan, N. (2009). Core verbal working-memory capacity: The limit in words retained without covert articulation. Quarterly Journal of Experimental Psychology, 62, 1420-1429.

Cordes, S., Gelman, R., Gallistel, C. R., \& Whalen, J. (2001). Variability signatures distinguish verbal from nonverbal counting for both large and small numbers. Psychonomic Bulletin \& Review, 8, 698-707.

Dehaene, S. (1992). Varieties of numerical abilities. Cognition, 44, 1-42.

Dehaene, S., Dehaene-Lambertz, G., \& Cohen, L. (1998). Abstract representations of numbers in the animal and human brain. Trends in Neurosciences, 21, 355-361.

Egeth, H. E., Leonard, C. J., \& Palomares, M. (2008). The role of attention in subitizing: Is the magical number 1? Visual Cognition, 16, 463-473.

Feigenson, L., \& Carey, S. (2003). Tracking individuals via object-files: Evidence from infants' manual search. Developmental Science, 6, $568-584$.

Feigenson, L., Dehaene, S., \& Spelke, E. S. (2004). Core systems of number. Trends in Cognitive Sciences, 8, 307-314.

Ferrand, L., Riggs, K. J., \& Castronovo, J. (2010). Subitizing in congenitally blind adults. Psychonomic Bulletin \& Review, 17, 840-845.

Fougnie, D., \& Marois, R. (2006). Distinct capacity limits for attention and working memory: Evidence from attentive tracking and visual working memory paradigms. Psychological Science, 17, 526-534.

Fougnie, D., \& Marois, R. (2009a). Attentive tracking disrupts feature binding in visual working memory. Visual Cognition, 17, 48-66.

Fougnie, D., \& Marois, R. (2009b). Dual-task interference in visual working memory: A limitation in storage capacity but not in encoding or retrieval. Attention, Perception, \& Psychophysics, 71, 1831-1841.

Franconeri, S. L., Jonathan, S. V., \& Scimeca, J. M. (2010). Tracking multiple objects is limited only by object spacing, not by speed, time, or capacity. Psychological Science, 21, 920-925.

Gallace, A., Tan, H. Z., \& Spence, C. (2006). Numerosity judgments for tactile stimuli distributed over the body surface. Perception, $35,247-266$. 
Gallistel, C. R. (2007). Commentary on Le Corre \& Carey. Cognition, 105, 439-445. doi:10.1016/j.cognition.2007.01.010

Gallistel, C. R., \& Gelman, R. (1992). Preverbal and verbal counting and computation. Cognition, 44, 43-74. doi:10.1016/0010-0277 (92) $90050-\mathrm{R}$

Gallistel, C. R., \& Gelman, R. (2000). Non-verbal numerical cognition: From reals to integers. Trends in Cognitive Sciences, 4, 59-65. doi:10.1016/S1364-6613(99)01424-2

Gelman, R., \& Gallistel, C. R. (1978). The child's understanding of number. Cambridge, MA: Harvard University Press.

Gibbon, J. (1977). Scalar expectancy theory and Weber's Law in animal timing. Psychological Review, 84, 279-325.

Haladjian, H. H., \& Pylyshyn, Z. W. (2011). Enumerating by pointing to locations: A new method for measuring the numerosity of visual object representations. Attention, Perception, \& Psychophysics, 73, 303-308.

Horowitz, T. S., Birnkrant, R. S., Fencsik, D. E., Tran, L., \& Wolfe, J. M. (2006). How do we track invisible objects? Psychonomic Bulletin \& Review, 13, 516-523. doi:10.3758/BF03193879

Horowitz, T. S., \& Cohen, M. A. (2010). Direction information in multiple object tracking is limited by a graded resource. Attention, Perception, \& Psychophysics, 72, 1765-1775.

Hulleman, J. (2005). The mathematics of multiple object tracking: From proportions correct to number of objects tracked. Vision Research, 45, 2298-2309.

Jevons, W. S. (1871). The power of numerical discrimination. Nature, 3, 281-282.

Kahneman, D., Treisman, A., \& Gibbs, B. J. (1992). The reviewing of object files: Object-specific integration of information. Cognitive Psychology, 24, 175-219. doi:10.1016/0010-0285(92)90007-O

Kaufman, E. L., Lord, M. W., Reese, T. W., \& Volkmann, J. (1949). The discrimination of visual number. American Journal of Psychology, 62, 498-525.

Klahr, D. (1973). Quantification processes. In W. G. Chase (Ed.), Visual information processing (pp. 3-34). New York: Academic Press.

Le Corre, M., \& Carey, S. (2007). One, two, three, four, nothing more: An investigation of the conceptual sources of the verbal counting principles. Cognition, 105, 395-438.

Leonard, C. J., \& Pylyshyn, Z. W. (2003). Measuring the attentional demand of multiple object tracking (MOT) [Abstract]. Journal of Vision, 3(9), 582a.

Mandler, G., \& Shebo, B. J. (1982). Subitizing: An analysis of its component processes. Journal of Experimental Psychology: General, 111, 1-22. doi:10.1037/0096-3445.111.1.1

Meck, W. H., \& Church, R. M. (1983). A mode control model of counting and timing processes. Journal of Experimental Psychology: Animal Behavior Processes, 9, 320-334.

Miller, G. A. (1956). The magical number seven plus or minus two: Some limits on our capacity for processing information. Psychological Review, 63, 81-97.

Moyer, R. S., \& Landauer, T. K. (1967). Time required for judgements of numerical inequality. Nature, 215, 1519-1520. doi:10.1038/ $2151519 \mathrm{a} 0$

Oksama, L., \& Hyönä, J. (2004). Is multiple object tracking carried out automatically by an early vision mechanism independent of higher-order cognition? An individual difference approach. Visual Cognition, 11, 631-671.

Olivers, C. N. L., \& Watson, D. G. (2008). Subitizing requires attention. Visual Cognition, 16, 439-462.

Piazza, M., Fumarola, A., Chinello, A., \& Melcher, D. (2011). Subitizing reflects visuo-spatial object individuation capacity. Cognition.
Plaisier, M. A., Bergmann Tiest, W. M., \& Kappers, A. M. L. (2009). One, two, three, many-Subitizing in active touch. Acta Psychologica, 131, 163-170.

Plaisier, M. A., \& Smeets, J. B. (2011). Haptic subitizing across the fingers. Attention, Perception, \& Psychophysics, 73, 1579-1585.

Pylyshyn, Z. (1989). The role of location indexes in spatial perception: A sketch of the FINST spatial-index model. Cognition, 32, 65-97. doi:10.1016/0010-0277(89)90014-0

Pylyshyn, Z. W. (1994). Some primitive mechanisms of spatial attention. Cognition, 50, 363-384.

Pylyshyn, Z. W. (2001). Visual indexes, preconceptual objects, and situated vision. Cognition, 80, 127-158. doi:10.1016/S00100277(00)00156-6

Pylyshyn, Z. W., \& Annan, V., Jr. (2006). Dynamics of target selection in multiple object tracking (MOT). Spatial Vision, 19, 485-504.

Pylyshyn, Z. W., Haladjian, H. H., King, C., \& Reilly, J. (2008). Selective nontarget inhibition in multiple object tracking. Visual Cognition, 16, 1011-1021.

Pylyshyn, Z. W., \& Storm, R. W. (1988). Tracking multiple independent targets: Evidence for a parallel tracking mechanism. Spatial Vision, 3, 179-197. doi:10.1163/156856888X00122

Revkin, S. K., Piazza, M., Izard, V., Cohen, L., \& Dehaene, S. (2008). Does subitizing reflect numerical estimation? Psychological Science, 19, 607-614.

Riggs, K. J., Ferrand, L., Lancelin, D., Fryziel, L., Dumur, G., \& Simpson, A. (2006). Subitizing in tactile perception. Psychological Science, 17, 271-272.

Scholl, B. J. (2009). What have we learned about attention from multiple-object tracking (and vice versa)? In D. Dedrick \& L. Trick (Eds.), Computation, cognition, and Pylyshyn (pp. 49-77). Cambridge, MA: MIT Press.

Tombu, M., \& Seiffert, A. E. (2008). Attentional costs in multiple-object tracking. Cognition, 108, 1-25. doi:10.1016/j.cognition.2007.12. 014

Trick, L. M., \& Pylyshyn, Z. W. (1989). Subitizing and the FINST spatial index model. Bulletin of the Psychonomic Society, 27, 490.

Trick, L. M., \& Pylyshyn, Z. W. (1993). What enumeration studies can show us about spatial attention: Evidence for limited capacity preattentive processing. Journal of Experimental Psychology: Human Perception and Performance, 19, 331-351. doi:10.1037/ 0096-1523.19.2.331

Trick, L. M., \& Pylyshyn, Z. W. (1994a). Cueing and counting: Does the position of the attentional focus affect enumeration? Visual Cognition, 1, 67-100. doi:10.1080/13506289408402294

Trick, L. M., \& Pylyshyn, Z. W. (1994b). Why are small and large numbers enumerated differently? A limited-capacity preattentive stage in vision. Psychological Review, 101, 80-102. doi:10.1037/ 0033-295X.101.1.80

van Oeffelen, M. P., \& Vos, P. G. (1982). A probabilistic model for the discrimination of visual number. Perception \& Psychophysics, 32, 163-170.

Vetter, P., Butterworth, B., \& Bahrami, B. (2008). Modulating attentional load affects numerosity estimation: Evidence against a pre-attentive subitizing mechanism. PLoS ONE, 3, e3269.

Whalen, J., Gallistel, C. R., \& Gelman, R. (1999). Nonverbal counting in humans: The psychophysics of number representation. Psychological Science, 10, 130-137.

Zhang, H., Xuan, Y., Fu, X., \& Pylyshyn, Z. W. (2010). Do objects in working memory compete with objects in perception? Visual Cognition, 18, 617-640. 